Utah State University

DigitalCommons@USU

\title{
Using the WSA Model to Test the Parker Spiral Approximation for SEP Event Magnetic Connections
}

\author{
S. W. Kahler \\ C. N. Arge \\ Air Force Research Laboratory \\ David Alan Smith \\ Utah State University
}

Follow this and additional works at: https://digitalcommons.usu.edu/phys_stures

Part of the Physics Commons

\section{Recommended Citation}

Kahler, S. W.; Arge, C. N.; and Smith, David Alan, "Using the WSA Model to Test the Parker Spiral Approximation for SEP Event Magnetic Connections" (2016). Physics Student Research. Paper 17. https://digitalcommons.usu.edu/phys_stures/17

This Article is brought to you for free and open access by the Physics Student Research at DigitalCommons@USU. It has been accepted for inclusion in Physics Student Research by an authorized administrator of DigitalCommons@USU. For more information, please contact digitalcommons@usu.edu.

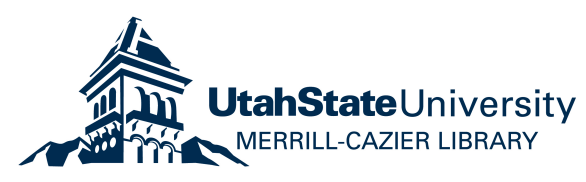




\title{
Using the WSA Model to Test the Parker Spiral Approximation for SEP Event Magnetic Connections
}

\author{
S.W. Kahler \\ C.N. Arge \\ Air Force Research Laboratory, Space Vehicles Directorate, 3550 Aberdeen Ave., Kirtland AFB, NM 87117, \\ USA \\ AFRL .RVB.PA@kirtland.af .mil \\ D.A. Smith \\ Utah State University, Logan, UT 84322, USA
}

\begin{abstract}
In studies of solar energetic $(E>10 \mathrm{MeV})$ particle (SEP) events the Parker spiral (PS) field approximation, based only on the measured 1 AU solar wind (SW) speed Vsw, is nearly always used to determine the coronal or photospheric source locations of the 1 AU magnetic fields. There is no objective way to validate that approximation, but here we seek guidelines for optimizing its application. We first review recent SEP studies showing the extensive use of the PS approximation with various assumptions about coronal and photospheric source fields. We then run the Wang-Sheeley-Arge (WSA) model over selected Carrington rotations (CRs) to track both the photospheric and 5 Rs source locations of the forecasted 1 AU SW, allowing us to compare those WSA sources with the PS sources inferred from the WSA Vsw forecast. We compile statistics of the longitude differences (WSA-PS) for all the CRs and discuss the limitations of using the WSA model to validate the PS approximation. Over nearly all of each CR the PS and WSA source longitudes agree to within several degrees. The agreement is poor only in the slow-fast SW interaction regions characterized by high-speed events (HSEs), where the longitude differences can reach several tens of degrees. This result implies that SEP studies should limit use of the PS approximation around HSEs and use magnetic field polarities as an additional check of solar source connections.
\end{abstract}

Subject headings: Energetic Particles - Acceleration, Magnetic fields - Models, Coronal Mass Ejections Low Coronal Signatures

\section{Introduction}

\subsection{Magnetic Solar Source Connections of SEP Events}

Studies of solar energetic $(E>1 \mathrm{MeV})$ particle (SEP) events have been limited by two factors. First, the events themselves are observed remotely from their solar sources and in situ, usually at 1 $\mathrm{AU}$ at the Lagrangian L1 point. Second, unlike photons or neutral particles, their propagation to $1 \mathrm{AU}$ is governed by magnetic field lines convected out from the Sun by the solar wind (SW) and characterized by turbulence and spreading (Ragot, 2011; Laitinen et al., 2013) in the interplanetary medium. The SEP primary acceleration sites are only broadly understood (Reames, 2013) as compact coronal regions of magnetic reconnection or shocks driven by coronal mass ejections (CMEs). 
A primary requirement in many SEP studies is to trace the in situ magnetic field of an observed SEP event back to its solar source through an estimate of its topology and geometry. This requirement is complicated by the complexity of closed and open coronal magnetic fields, which are typically represented with potential field source surface (PFSS) models that connect observed photospheric fields to a source surface at 2.5 Rs at which the fields are all radial from the Sun (Owens and Forsyth, 2013).

\subsection{The PS Model in SEP Studies}

The simplest assumption for the magnetic field source location is that it lies at the base of a Parker spiral (PS) field (Owens and Forsyth, 2013) defined by a constant radial flow of the SW to L1 with some average SW speed Vsw. This assumption restricts the solar source latitude to lie in the ecliptic plane, with a resulting heliographic latitude range of $\pm 7.25^{\circ}$, depending on the solar $\mathrm{B}_{0}$ angle. Fixed Vsw values were invoked in recent statistical studies of coronal holes $(\mathrm{CHs})$ and SEP events (W60, Shen et al., 2006; 2010) and of longitudinal distributions of SEP events $(\mathrm{Vsw}=450$ $\mathrm{km} \mathrm{s}^{-1}$, Lario et al., 2006; Vsw $=400 \mathrm{~km} \mathrm{~s}^{-1}$, Lario et al., 2013).

In general, however, most work to relate SEP events to their solar sources begins with the PS approximation using the local in situ Vsw. For some recent SEP studies, that inferred source location was taken as the magnetic footpoint, with no further qualification, and then compared to the location of a solar event, usually a flare. This approach was used in STEREO A and B (STA, STB) observations of the 17 January 2010 (Dresing et al., 2012), 3 November 2011 (Prise et al., 2014) and 11 April 2013 (Cohen et al., 2014) SEP events, ${ }^{3}$ He-rich events (Wiedenbeck et al., 2013), and in statistical studies of $E>50 \mathrm{keV}$ electron events (Dresing et al., 2014; Agueda et al., 2014). Similarly, Rouillard et al. (2012) accounted for the 21 March 2011 SEP onset times at STA and L1 by matching PS footpoints with an EUV wave and the edges of CME-driven streamer deflections. In their comparisons of SEP events first with flares and CMEs and then with EIT waves, Miteva et al. (2013, 2014, respectively) acknowledged that their PS extrapolations were valid only to a $2.5 \mathrm{Rs}$ source surface, but used those locations as the 1
AU footpoints in their work. Klassen et al. (2015) found that the PS magnetic separation of the footpoints of STA and STB on the PFSS source surface was $47^{\circ}$ for a spike electron event observed at both spacecraft.

\subsection{The PS+PFSS Model in SEP Studies}

\subsubsection{Photospheric Sources}

Other recent SEP event analyses have combined the PS approximation with the PFSS model (PS+PFSS) to determine the photospheric source footpoints. In the PFSS model the open photospheric fields diverge to the overlying source surface at $2.5 \mathrm{Rs}$, or at $5 \mathrm{Rs}$ in the coupled PFSS+SCS (SCS, Schatten (1972) current sheet) model approach of this work, as shown in the three Carrington rotations (CRs) of Figure 1. The limitations of the PFSS model due to appropriate photospheric field maps have been addressed by various authors (Luhmann et al., 2008; Nitta and DeRosa, 2008; Linker et al., 2013). Wiedenbeck et al. (2013) surveyed clusters of field lines that expand from small photospheric sources of angular radii $\mathrm{R}$ to the overlying longitudinal extents on the $2.5 \mathrm{Rs}$ source surface. They selected the largest longitudinal extents of field clusters for each day from $\sim 5100$ PFSS daily maps as a function of R. Their plot, reproduced as Figure 2, shows that these largest extents are several tens of degrees. Thus, if the PS extrapolation lands in the appropriate cluster, the photospheric location is correct to within the size of $\mathrm{R}$, but for an incorrect cluster, the inferred photospheric location is incorrect by tens of degrees.

The PS+PFSS approximation is used to make photospheric field connections to SEP events. Klein et al. (2008) used PS+PFSS to connect L1 footpoints to open fields in active regions for seven simple SEP events. Ko et al. (2013) similarly correlated SEP Fe/O abundances with the footpoint field strengths for 24 gradual SEP events. In various STA/STB SEP event comparisons with EUV waves, Nitta (2012), Park et al. (2013, 2015), and Gómez-Herrero et al. (2015) also followed this approach.

The question of how accurately the PS or the PS+PFSS approximation selects the source longitude is rarely considered, but Klein et al. (2008) cited two basic uncertainties to infer an error range 
of less than $\pm 10^{\circ}$. First, Nolte \& Roelof (1973) in their introduction of the PS method (called the EQRH approximation) argued that the transition from quasi-rigid rotation to radial flow at heights above the source surface and the acceleration of solar wind with distance were offsetting factors which combined to produce an uncertainty of $<10^{\circ}$. The second uncertainty was that of cross-field displacement of flux tubes resulting from interplanetary magnetic fluctuations. Klein et al. (2008) cited a value of $\pm 6^{\circ}-10^{\circ}$, but recent calculations (Ragot, 2011; 2012) show flux tube displacements from the nominal PS field may be as high as several tens of degrees in very turbulent fields. In addition, Kahler et al. (2014) did a direct comparison of PS and WSA (Sec. 2.1) models for 33 SEP events and found average differences of $\sim 20^{\circ}$ for both photospheric source latitudes and longitudes. While the resulting longitudinal uncertainty $\Delta \mathrm{L}$ of the PS method is difficult to estimate, the claim of $\pm 10^{\circ}$ may be a low estimate.

\subsection{2. ${ }^{3}$ He-rich SEP Events as PS Tests}

Impulsive ${ }^{3} \mathrm{He}$-rich events provide opportunities for testing the calculated PS+PFSS connections to assumed known compact solar SEP sources. MacNeice et al. (2011) used a stringently selected list of 15 impulsive SEP events with well determined solar source regions for a comparison of the PS model, with and without the PFSS model, against the WSA and WSA-ENLIL models. The average longitudinal errors in footpoint location for both WSA and WSA-ENLIL (Odstrcil and Pizzo, 2009) models were $\simeq 20^{\circ}$ and comparable to the $\mathrm{PS}$ and PS+PFSS averages of $23^{\circ}$ and $25^{\circ}$.

Data from the Atmospheric Imaging Assembly (AIA; Lemen et al., 2012) on the Solar Dynamics Observatory (SDO) have allowed observers to determine ${ }^{3} \mathrm{He}$-rich event source regions with a certainty not previously available (MacNeice et al., 2011). Bučík et al. (2014) examined four ${ }^{3} \mathrm{He}-$ rich events observed in July 2011 at STA, STB and L1, using the PS+PFSS model to determine the footpoints. The two candidate source regions AR 11244 and AR 11246 were separated by about $50^{\circ}$ in longitude, with a similar width for each of their field line extensions to the 2.5 Rs source surface. The PS+PFSS model source fields matched the presumed source ARs in all four cases, but in two cases the PS field line lay near the boundaries of the two broad source surface extensions of the ARs. Chen et al. (2015) did a similar analysis of two pairs of ${ }^{3} \mathrm{He}$-rich events observed at L1 and also found PS footpoints within extended magnetic connections to the source ARs of each pair. A recent unusual series of ${ }^{3} \mathrm{He}$-rich events observed on STA and STB with a longitudinal separation of $38^{\circ}$ in May 2014 was due to a source region with a $95^{\circ}$ width on the source surface (Wiedenbeck et al., 2015). These results support the concern of MacNeice et al. (2011) that impulsive SEPs may escape the Sun along field lines extending well away from the compact acceleration sites, undermining the validity of ${ }^{3} \mathrm{He}$ event sources as compact targets for tracing field lines.

A recent survey of $26{ }^{3} \mathrm{He}$-rich event periods at L1 by Nitta et al. (2015) produced less favorable results. They found a large longitudinal spread of SEP source regions extending well beyond both the simple PS and the PS+PFSS source regions. In addition, they determined whether the PS+PFSS SEP source ARs had any open field regions matching the L1 field polarities. In 10 cases, including 3 of the 4 events of the Chen et al. (2015) study, there were no matching open field polarities to account for source connections. Their results confirmed the conclusion of Wiedenbeck et al. (2013) that within the PFSS model the longitudinal extents of field lines along the heliographic equator can not account for the large angular ranges of some ${ }^{3} \mathrm{He}$-rich events.

\subsection{Beyond PS+PFSS}

Several SEP event studies have moved beyond the straightforward PS or PS+PFSS approach to solar connections. Luhmann et al. (2012) studied the series of SEP events in August 2010 observed at L1 and STB by combining a PFSS and ENLIL/cone model to describe the timedependent perturbations of the CMEs on the interplanetary fields. In their SEPMOD model, SEPs were injected at the $\sim \mathbf{2 0} \mathbf{R s}$ inner boundary of ENLIL over regions of open fields extending from flaring regions. Injection rates were based on assumed flare-related parameters and reproduced the major features of the observed SEP events. Examining the EUV wave locations in the 11 April 2013 SEP event, Lario et al. (2014) compared source locations for L1, STA, and STB derived from three methods: PS only; PS with the 
Magnetohydrodynamics Outside a Sphere (MAS) model (Riley and Lionello, 2011) to $30 \mathrm{Rs}$; and PS+PFSS. The PS latitudes, restricted to \pm $7^{\circ}$, disagreed with the MAS and PS+PFSS latitudes by up to $19^{\circ}$. The three methods were in good agreement in longitude for L1 $\left(186^{\circ}-187^{\circ}\right)$ and STA $\left(295^{\circ}-302^{\circ}\right)$, but ranged from $35^{\circ}$ to $55^{\circ}$ for STB. Note that the three methods all depend on the initial PS assumption.

Rouillard et al. (2011) determined the times when L1 and STB were first magnetically connected to a CME-driven shock on 3 April 2010. They used an ENLIL model of the CME observations and in situ shock observations at $1 \mathrm{AU}$ to validate the model. They also modified the model upstream Vsw values to match the observed in situ values at STB and L1 over the five-day period preceding shock arrival at $1 \mathrm{AU}$ to get optimum PS connections to the shock. The 12-hour delay between CME eruption and L1 SEP onset was consistent with the time for L1 connection to the western edge of the shock.

These works indicate a trend toward increasingly spatially and temporally resolved properties of coronal shocks and particle acceleration that can only be tested at L1 with accurate determinations of solar magnetic connections. The PS approximation widely used in SEP studies assumes a completely ballistic Vsw with no stream-stream interactions which could distort the PS configuration to result in footpoint longitude displacements. The WSA model includes these interactions in an adhoc manner to produce a forecast $\mathrm{Vsw}$ at L1. In the next section we describe the model and its validation and apply the model to a number of CRs. The model is expanded to include the solar source longitudes and latitudes of the calculated L1 SW. The goal is to compare the WSA source longitudes with their corresponding PS longitudes deduced from the $\mathrm{L} 1$ values of the WSA forecasted Vsw. This procedure will test the limits of the PS method, using only results of the WSA model. Alternative comparisons between (1) PS source locations calculated with L1 spacecraft observations of Vsw and (2) the WSA model outputs for the same L1 solar sources will be done elsewhere.

\section{Data Selection and Analysis}

\subsection{The WSA Model for SEP Event Solar Sources}

Here we use the Wang-Sheeley-Arge (WSA) model (Arge et al., 2004, MacNeice, 2009; Norquist and Meeks, 2010) to compare the coronal and photospheric source regions of the model with those of the PS approximation. WSA is a combined empirical and physics-based representation of the corona and quasi-steady global SW flow. Briefly, a standard PFSS model is used here with a National Solar Observatory (NSO) magnetogram from the Global Oscillation Network Group (GONG) to generate an open radial magnetic field on the PFSS surface at $2.5 \mathrm{Rs}$, which in turn serves as an inner boundary for the SCS model (MacNeice, 2009) that provides a nearly radial field at 5 Rs with a thin current sheet. Then a $1 \mathrm{D}$ kinematic model with an ad-hoc model to account for stream interactions is used to forecast Vsw and the IMF polarity at L1. When the coronal portion of WSA is used to drive an advanced MHD model like ENLIL, the SCS model within WSA is extended out to $21.5 \mathrm{Rs}$ (Lee et al., 2013).

For each CR since 2009, magnetograms served as input to the Air Force Data Assimilative Flux Transport (ADAPT) model, which makes use of rigorous data assimilation methodologies (Hickmann et al., 2015). That model generates for a given day a flux-evolved synchronic map, or set of maps based on several realizations of the flux transport parameters (Arge et al., 2013). The WSA model was run on synchronic ADAPT maps for a given day of a $\mathrm{CR}$. The maps are available at ftp://gong2.nso.edu/adapt/maps/public/gong/.

WSA forecasts are generated by letting the SW packets leave the Sun from the 5 Rs outer boundary with a fixed speed for each packet and a uniform cadence of one packet every $2.5^{\circ}$ of solar longitude. The SW portion of the model is a simple 1D modified kinematic model in which the wind packets interact every $1 / 8 \mathrm{AU}$ to allow interaction between faster and slower packets (MacNeice et al. 2011). The model propagates SW parcels out to L1 (or any other desired point in space), keeping track of their source longitudes back at the Sun along with other parameters such as the magnetic field strengths and polarities of the footpoints. 
The interactions result in nonuniform packet arrival times at $\mathrm{L} 1$, but each packet is tagged with a WSA forecast value of $\mathrm{Vsw}_{\mathrm{Sw}}$ and $\mathrm{B}$ and a direct mapping of its magnetic field line back to its source region. We compared the WSA sources with the PS sources calculated for each forecast value of Vsw.

Kahler et al. (2014) used the WSA model in this mode to look for angular separations between L1 magnetic footpoints and sources of CMEs associated with SEP events. They used synoptic maps from Mount Wilson Observatory (MWO) and the NSO and compared WSA forecasts with L1 observations of $\mathrm{Vsw}$ and $\mathrm{B}$. Here we use ADAPT maps derived from NSO full disk magnetograms, and we are not concerned with their forecast accuracy, but rather with how the 1 and 5 Rs source footpoints compare with those deduced from the PS approximation.

\subsection{Selection of CRs}

To compare the 5 Rs solar source longitudes of the WSA ADAPT (hereafter WSA) model (Hickman et al., 2015) with those derived from the PS approximation, we first sought WSA model prediction runs for full CRs, including some periods of high solar activity. To include SEP events, we used the Richardson et al. (2014) list of $E>25$ $\mathrm{MeV}$ SEP events observed at L1 and STEREO A/B. Those active periods of SEP events are also characterized by interplanetary CMEs (ICMEs) for which the WSA model is not appropriate, so we further selected only CRs with no more than four ICMEs on the list of Near-Earth ICMEs compiled by Richardson and Cane (2012) at http://www.srl.caltech.edu/ACE/ASC/DATA/ level3/icmetable2.htm. For each $\mathrm{CR}$ we selected three ADAPT maps corresponding to times near the beginning, middle and end of that $\mathrm{CR}$ and compared the WSA model forecast Vsw based on that day with the Vsw observed at the ACE satellite to look for qualitative agreement between the two Vsw profiles.

ADAPT is a data assimilative ensemble model providing 12 different realizations for any given moment in time. Each set of 12 ADAPT realizations was comprised of three subsets of four maps having fixed meridional flow rates but randomly varying super granule patterns. From the 36 available WSA runs (12 realizations $\times 3$ different days) the one judged to have the best qualitative match to the observed Vsw profile for a given $\mathrm{CR}$ was used in our analysis. Figure 3 shows superposed WSA and ACE Vsw plots for three sample CRs. The plots are similar to those illustrated in Arge et al. (2004) and in MacNeice (2009). For forecasting and validation studies the irregular time sequence of the WSA forecasts is converted to a uniform sequence of $\sim 5.3$ data points per day by interpolating values of the original WSA forecasts. The resulting plots of Figure 3 belie the original nonuniform sequences of the WSA model at L1.

The 21 selected CRs are listed in Table 1, along with their start dates and the numbers of SEP events and ICMEs (column 3). Note that the first WSA forecast values of Vsw occur several days after the start of each CR due to the time for SW propagation time to L1. The portions of the WSA profiles extending beyond the ends of the CRs into the subsequent CRs are not shown and were not used in the analysis. The Vsw profiles are characterized by rapid increases to high speeds followed by the longer gradual declines due to the decreasing slower wind inputs to lower speeds. The rapid Vsw increases have been termed high-speed events (HSEs), defined by Owens et al. (2008) as increases of $\Delta \mathrm{Vsw} \geq 100 \mathrm{~km} \mathrm{~s}^{-1}$ in 2 days or less. The WSA forecasts of HSEs have been validated by Owens et al. (2008), by MacNeice (2009) with a more complex HSE definition, and with both definitions by Norquist (2013), who concluded that the WSA generally overpredicts the occurrence of HSEs, but often underpredicts because of the occurrence of ICMEs. We note that the only ICME occurrence among the three CRs of Figure 3 occurred on 1617 May 2012 of CR 2123. We did not attempt to separate ICMEs from HSEs in our analysis. The goal was to compare the WSA source longitudes with the PS longitudes on the assumption that the WSA model provides a realistic calculation of both source longitude and Vsw. The question then was to determine the differences between the WSA and the inferred PS longitudes.

\subsection{Overview and Declining Vsw Regions}

For each CR we calculated and compared the 5 Rs running values of the source Carrington longitudes (CLs) of the WSA and of the PS approximation based on the WSA forecast of Vsw. Figure 4 shows PS and WSA CL connections for three CRs, 
each beginning with $360^{\circ}$ for the earliest CR date and progressing monotonically down to near $0^{\circ}$, where we have cut off the Vsw values before the beginning of the subsequent CR. The WSA model generates $360^{\circ} / 2.5^{\circ}=144$ data points per CR, but the variable Vsw at 5 Rs produces an irregular cadence of those WSA model data points at L1 that contrasts with the replotted regular cadence of the Figure 3 plots, as discussed above.

The PS and WSA CL connections agree so well over nearly all the CL ranges that only the overplotted WSA data points are visible. The agreement is best in the periods of declining high Vsw, as expected for solar source regions of progressively slower speeds characterized by ballistic flows in rarefaction regions with no stream-stream interactions, indicated in the middle region of Figure 5, the iconic cartoon of Richardson (2004). However, if Vsw declines sufficiently rapidly, one encounters PS periods discussed by Nolte et al. (1977) and referred to as "dwells", in which the PS CLs are nearly constant for one to three days. Their interpretation of those periods, assuming the PS approximation, was that the SW was emitted from constant CLs, but from varying coronal heights. Examples of dwells in Figure 4 are seen at rotation days 19-20 and 26-27 of CR 2100, 9-11 of CR 2126, and 26-27 of CR 2154.

The WSA model assumes radial flows at 5 Rs and no crossing of stream flows in the 2-D plane of the ecliptic. The latter condition sets a basic limit on the WSA modeled values of $\mathrm{dVsw} / \mathrm{dt}$. When Vsw is decreasing (the rarefaction region of Figure 5), the PS footpoint longitude relative to central meridian, $\phi$, moves westward at a rate that depends on $\mathrm{dVsw} / \mathrm{dt}$. With a sufficiently large $-\mathrm{dVsw} / \mathrm{dt}$, we may find that $\mathrm{d} \phi / \mathrm{dt}$ matches the solar rotation rate and the $\mathrm{CL}$ will appear to be fixed over an extended time period. Taking

$$
\mathrm{d} \phi / \mathrm{dt}=\mathrm{D} \Omega\left((\mathrm{Vsw})^{-2}(\mathrm{dVsw}) / \mathrm{dt}\right)>\Omega,
$$

where $\Omega$ is the solar rotation rate and $\mathrm{D}$ is $1 \mathrm{AU}$, then if

$$
-\mathrm{d}(\mathrm{Vsw}) / \mathrm{dt}>(\mathrm{Vsw})^{2} / \mathrm{D}
$$

we encounter the unphysical situation that earlier arriving faster SW has left the Sun after the later arriving slower solar wind, requiring the fast and slow streams to cross each other in the ecliptic plane (Riley and Lionello, 2011), contrary to the WSA model. We do not encounter this situation either in the L1 observations or in the WSA Vsw profiles. Note that the WSA L1 data points are widely spaced in time, indicating long intervals between plasma arriving from sources uniformly spaced $2.5^{\circ}$ apart. However, the agreement of the PS with the output of the WSA model, with its assumed radial SW flows at 5 Rs, tells us that the dwells encountered in the PS approximation may not be so much artifacts as real source regions of limited CL ranges.

\subsection{The Compression Regions of Vsw}

The only times of significant disagreement between WSA and PS CLs occur at the relatively low values of Vsw preceding HSEs. These periods are characterized by WSA plasmas from multiple solar source regions separated by $2.5^{\circ}$ but with closely bunched arrival times at L1 produced by the WSA model. They correspond to the slow SW regions at the leading edges of the compression or corotating interaction regions (CIRs) of Figure 5. The slowly varying Vsw yields a relatively fixed PS CL during the bunched times, but as the WSA CLs move eastward in these time bunches, the CL difference $\Delta \mathrm{L}=\mathrm{WSA}-\mathrm{PS}$ becomes significantly negative. These negative $\Delta \mathrm{L}$ excursions are not so obvious on the time plots of Figure 4, but they are clear in the CR scatter plots of $\Delta \mathrm{L}$ versus $\mathrm{Vsw}$ in Figure 6 for the data points of the same three CRs of Figure 4. Most WSA points lie along the line $\Delta \mathrm{L} \sim 0^{\circ}$, but the several columns of points are the large deviations corresponding to the slow Vsw values at bunches preceding the HSEs. We use these plots to define our version of HSEs and select those cases in which the lowest $\Delta \mathrm{L} \leq-15^{\circ}$. There are then 9 HSEs for the three CRs of Figure 6 and a total of 57 such HSEs in our 21 CRs. We give the $\Delta \mathrm{Vsw}$ of each HSE and the preceding $\Delta \mathrm{L}$ in columns 4 and 5 of Table 1 .

In the top left panel of Figure 7 we mark the four CR 2126 HSEs of Table 1 and show in the other three panels how $\Delta \mathrm{L}$ decreases through the sequence of WSA model data points. Note that the sequence of WSA data points, rather than time, constitutes the independent variable. In each sequence the negative $\Delta \mathrm{L}$ values accumulate 
only through the constant $\mathrm{Vsw}(\Delta \mathrm{Vsw}=0)$ values in a data point bunch. $\Delta \mathrm{L}$ resets to $0^{\circ}$ with a subsequent increase or decrease of Vsw. The largest $\Delta \mathrm{L}$ values of all but one of the 59 HSEs of Table 1 occurred just before a large increase of Vsw, similar to the HSE plots for the other CRs.

The 57 cases of $\Delta \mathrm{L} \leq-15^{\circ}$ of Table 1 are plotted against their corresponding preceding speeds $\mathrm{V}_{\mathrm{sw}}$ and $\Delta \mathrm{Vsw}$ values in Figure 8 . The maximum $\Delta \mathrm{L}$ values are weighted strongly toward both low Vsw and large $\Delta \mathrm{Vsw}$, as seen in the example of Figure 7. All values of $\Delta \mathrm{Vsw}$ exceed the $100 \mathrm{~km}$ $\mathrm{s}^{-1}$ threshold for the original HSE definition of Owens et al. (2008). Nearly all the largest $\Delta \mathrm{L}$ excursions lie in the $-30^{\circ}$ to $-40^{\circ}$ range, which we take as the maximum range of difference resulting from the use of the PS approximation. $\Delta \mathrm{L}$ is strongly correlated $(\mathrm{P}<0.001)$ with $\Delta$ Vsw (C.C. $=-0.49$ ) and Vsw (C.C. $=0.53)$, but the large scatter suggests that the extreme $\Delta \mathrm{L}$ values can be reached for almost any HSEs if we assume the $\Delta \mathrm{Vsw}_{\mathrm{sw}}>100 \mathrm{~km} \mathrm{~s}^{-1}$ criterion. In no case in our comparison did we find positive values of $\Delta \mathrm{L}$ exceeding $1^{\circ}$, so the PS approximation CL was never significantly west of the WSA CL for the assumed 5 Rs sources.

\subsection{The HSE Magnetic Polarities}

The WSA program tracks the source magnetic polarities as well as the CLs. The generally close agreement between the WSA and PS CLs evident for most of each CR (Figure 4) ensures that both source regions will have the same magnetic polarity. However, for the periods preceding the HSEs the large $\Delta \mathrm{L}$ values (Figure 8) suggest that the PS CLs displaced westward of the WSA CLs may lie in opposite polarity photospheric source regions, as can be seen in Figure 1. We examined the magnetic polarities across the $\Delta \mathrm{Vsw}=0$ data bunches preceding the HSEs and list them in the last column of Table 1. Those polarities changed in 11 of the 57 HSEs throughout the constant Vsw periods, and in each case the polarity change occurred at the start of a series of non-zero $\Delta \mathrm{Vsw}$, as shown in the Figure 9 examples.

Polarity changes can also occur for $\Delta \mathrm{L}$ excursions smaller than the $\leq 15^{\circ}$ associated with HSEs. Figure 9 shows that the selected $\Delta \mathrm{L}$ excursions also occurred during low values of $\Delta \mathrm{Vsw}$. WSA sources of the long data-point sequences of $\Delta \mathrm{Vsw}$
$=0$ correspond to slow wind regions (Figure 7 ) that map back to the western edges of coronal hole extensions to 5 Rs. The subsequent HSEs then map back to the high-speed regions of the coronal hole interiors, as expected in the WSA model.

\subsection{Photospheric Footpoint Connections}

Our WSA-PS footpoint analysis thus far has been restricted to comparisons at the $5 \mathrm{Rs}$ source surface. This may be appropriate for some SEP event analyses, but we can extend the comparison to the photospheric footpoint longitudes CLp using the basic PFSS+SCS approximation with the NSO magnetograms shown in Figure 1. The magnetic field line sources at the 5 Rs source surface generally converge to smaller coronal hole $(\mathrm{CH})$ sources at $1 \mathrm{Rs}$, which is most evident in the CRs 2135 and 2142. We expect that if the WSA and PS CLs share the same CH source, then the photospheric WSA-PS $\Delta \mathrm{Lp}$ should be less than the corresponding $\Delta \mathrm{L}$ at 5 Rs. On the other hand, when the WSA and PS 1 Rs sources lie in different $\mathrm{CHs}$, the resulting $\Delta \mathrm{Lp}$ values will usually be much larger.

In Figures 10 and 11 we show the CLp values for two CRs, plotted as functions both of WSA data points (top) and of time (bottom). Similar to the plots of the $5 \mathrm{Rs}$ CLs in Figure 4, the data point sequence of $\mathrm{Lp}$ begins several days after the CR start and extends to the end of the CR. We saw earlier that at the bunches of WSA data points the PS CL remains nearly constant while the WSA CLs move eastward, and those cases are seen in the flat extensions of the fixed PS CLp after the WSA longitudes progress to lower CLps in the top panels. The WSA bunches occur at the sharp drops in CL corresponding to the boundaries of the source $\mathrm{CHs}$, where the source magnetic polarities may or may not change. For our purpose of diagnosing the probability that the PS CLp approximation matches the WSA CLp model at $1 \mathrm{Rs}$, the time sequences in the bottom figure panels are the definitive comparisons. As we saw with the 5 Rs comparisons of Figure 4, the significant $\Delta \mathrm{Lp}$ differences are a minor aspect of the CRs and occur just preceding the HSEs evident in the Vsw profiles. However, the top panels of Figures 10 and 11 show that when they occur, those CLp differences can reach several tens of degrees.

We further find that there is little correlation 
between the $\Delta \mathrm{L}$ and $\Delta \mathrm{Lp}$ values, again as expected from the extensions to $5 \mathrm{Rs}$ of the $1 \mathrm{Rs}$ field sources shown in Figure 1. Large $\Delta \mathrm{L}$ values may be associated with small $\Delta \mathrm{Lp}$ values when the WSA and PS sources originate from the same $\mathrm{CH}$, but with different $\mathrm{CH}$ sources small $\Delta \mathrm{L}$ can be associated with large $\Delta \mathrm{Lp}$. In Figure 12 we compare histograms of logs of the numbers of $\Delta \mathrm{L}$ and $\Delta \mathrm{Lp}$ for all $21 \mathrm{CRs}$ and find that $\Delta \mathrm{Lp}$ extends to about twice the angular range of $\Delta \mathrm{L}$, but is more concentrated at $\Delta \mathrm{Lp} \sim 0$ than is $\Delta \mathrm{L}$. Qualitatively this means that if both the WSA and PS footpoints are within several 10s of degrees at 5 Rs, they will be close together at the source $\mathrm{CH}$ CLs.

\section{Summary of Results}

In this work we started with the WSA SW model incorporating solar magnetic field evolution into a coupled PFSS+SCS model with an outer boundary set at $5 \mathrm{Rs}$ combined with simple interplanetary dynamics. WSA yields L1 values of Vsw and magnetic field polarity along with the 1 Rs and 5 Rs CLs of each arriving SW packet. We use those Vsw values to track the $\Delta \mathrm{L}$ between the WSA and PS CLs as the basis for a comparison with the PS model. WSA should match the actual SW better than the PS approximation, since it takes into account stream interactions. In contrast to previous studies, this comparison is done completely within the context of the WSA model and uses no in situ spacecraft observations. The goal is to determine when and how well the PS approximation agrees with WSA. We then apply to spacecraft observations of Vsw the lessons learned from the WSA model comparisons to determine the validity of the PS approximation.

To first order, the results shown in Figure 4 indicate that the PS approximation yields excellent agreement with the WSA CLs. The very nonuniform cadence of the bunches of SW parcels at L1 shown in Figures 7 and 9 is obviously an artifact of the limited spatial and temporal resolution of the WSA model, but it has allowed us to examine the physics leading to the largest $\Delta \mathrm{L}$ and $\Delta \mathrm{Lp}$ values. The SW of the declining Vsw profiles of the WSA seems to propagate nearly ballistically from the eastern and central regions of $\mathrm{CHs}$ and undergoes minimal stream-stream interactions, so that the PS assumption works very well in that situation. The times of poor PS agreement with WSA appear only when the slow SW on the leading edges and the fast SW of the trailing edges of the stream interaction regions results in WSA CLs displaced eastward from the inferred PS CLs. The $\Delta \mathrm{L}$ immediately resets to 0 at the onset of HSEs (Figures 7 and 9), but this again is likely a misleading artifact of the WSA. The periods of poor PS approximation should probably be taken as those of both the slow and fast Vsw around the HSEs. Although these significant displacements constitute a large fraction of the WSA SW packets, they are only a small fraction of the duration of each CR for both 5 Rs and 1 Rs, as evident in Figures 4, 10 and 11.

The $\Delta \mathrm{L}$ differences statistically increase with lower pre-HSE SW speeds and larger $\Delta \mathrm{Vsw}$ speeds, as shown in Figure 8. We suggest therefore that the periods around the larger increases in Vsw at L1 spacecraft data will be the times when the PS approximation is poorest, and that the inferred PS CL will be improperly displaced to the west because stream interactions are not taken into account. We also sought to establish upper limits to the magnitudes of $\Delta \mathrm{L}$ and $\Delta \mathrm{Lp}$, and statistically these are $\sim-35^{\circ}$ and $-80^{\circ}$, respectively, as shown in Figure 12, the log plots of those distributions. The more peaked distribution near $0^{\circ}$ of the bottom photospheric plot shows qualitatively that one is more likely to get a good approximation to the photospheric footpoint, useful for comparisons of SEP events with EIT waves, than to the 5 Rs source surface footpoint, more applicable to CME shock studies. Although a direct quantitative comparison is not possible, the $\Delta \mathrm{Lp}$ distribution of the lower panel of Figure 12 appears at least consistent with the Figure 2 (Wiedenbeck et al. 2013) broad angular distributions of the photospheric source fields on the PFSS source surface.

\section{Further Considerations of the PS Ap- proximation}

We have seen in Section 1 that the determination of solar CLs of magnetic fields is an essential step to relate SEP events at L1 to their solar injection sources. The PS approximation has been extensively used, but it has not previously been critically examined except for studies with 
impulsive SEP events. Those event comparisons were not encouraging, but the simplicity of the PS approximation using SW observations at L1 ensures its continued use to determine SEP source regions. The basic problem, especially for gradual SEP events associated with CMEs, is that we have no absolute knowledge of the solar source regions of L1 field lines to use as tests of the PS approximation. A further complication is that the coronal fields observed at the times of SEP events have evolved from the fields of 3-4 days earlier from which the observed L1 SW originated. The problem for the investigator is to determine when and how well the PS approximation might provide a credible SEP source region.

The uncertainty of the source surface footpoint locations inferred from the PS approximation alone is difficult to determine, but the requirement of matching source and in situ magnetic field polarities provides a valuable check on PS approximations. The in situ field polarities are usually unambiguous at L1 when determined from both field orientations and heat-flux flow directions (Owens and Forsyth, 2013). We reported in Section 2.5 that the WSA source magnetic polarities were reversed in 11 of the 57 HSEs (Table 1) when the PS approximation was used. Since our HSEs were defined in terms of the largest $\Delta \mathrm{L}$ values we encountered in the 21 CRs of the study, they constitute a worst case situation for polarity mismatches from the PS approximation. The relative numbers of mismatches encountered may be small, but they still should provide a necessary tool to be used with the PS approximation.

Riley and Lionello (2011) considered the errors resulting from "ballistic" (equivalent to the PS) SW mappings between 30 Rs to 1 AU. They anticipated qualitatively that the rarefaction regions would map reasonably well, and the compression, or interaction, regions would be distorted as the preceding slow SW is accelerated and the trailing fast SW is decelerated. This implies that the PS approximation in the interaction regions would place the slow SW CLs too far to the west and the fast SW CLs too far to the east. In the WSA model, however, the ad hoc interaction of slow and fast SW produces the periods of slow SW bunching of L1 data points that precede HSEs (Figure $7)$. Throughout these bunches Vsw is nearly constant as the WSA CLs track progressively east- ward and away from the fixed PS CLs, as indicated in Figures 7 and 9. The bunching regions therefore appear to be predominately fast SW regions considerably decelerated by the preceding slow SW regions, resulting in WSA CLs displaced eastward of the PS CLs. The sharp HSEs characteristic of the WSA Vsw profiles contrast with the smoothed boundaries of the Vsw stream profiles produced by a more realistic heliospheric MHD model ( $\mathrm{Ri}-$ ley and Lionello, 2011) that reduces SW gradients. Thus the $\Delta \mathrm{L}$ excursions shown in Figure 12 should be considered upper limits to those produced by more realistic models.

We must keep in mind the limitations of the WSA model as we compare the PS approximation with the model outputs. The SW parcels are projected radially with fixed speeds and no SW acceleration term. The nonlinear dynamic interactions of different SW streams near the Sun are different from those near L1 (Riley and Lionello, 2011), providing another limitation on models of kinematic evolution, such as the WSA model used here. We anticipate that a similar comparison of PS against the more advanced WSA-ENLIL model (MacNeice et al., 2011), which includes solar wind dynamics, could yield CL results inconsistent with those presented here. Another big factor is the presence of ICMEs, which are not part of the WSA model, and can considerably distort the magnetic geometry of the evolutionary SW.

Thus far we have limited our consideration of determining field line sources to the use of $1 \mathrm{AU}$ observations of Vsw, perhaps supplemented with $\mathrm{B}$ field polarities, to use in the PS approximation. Several alternative techniques using additional 1 AU SW observations have been proposed, which could also be useful for determining solar source fields in SEP events. Schulte in den Bäumen et al. (2012) and Li et al. (2016) add to the 1 AU $\mathrm{Vsw}$ values averages of the $1 \mathrm{AU}$ radial and azimuthal B field components to map the interplanetary B field lines throughout the heliospheric ecliptic plane. Their model allows field lines to cross SW streams of different Vsw values, can detect loops, and permits azimuthal field components at the solar source region of choice. They supplement the B field and Vsw observations with accompanying suprathermal pitch angle distributions to confirm the field topologies.

Jackson et al. (2015) combine latitudinal field 
components at the 1.6 Rs cusp surface of the current-sheet source surface (CSSS) model and global velocities inferred from interplanetary scintillation (IPS) observations to model the $1 \mathrm{AU}$ Bn components. They had in mind closed loops as the sources of cusp-surface latitudinal fields, but open nonradial fields are a more likely alternative. A related idea (Manoharan et al., 2014) is to use velocities from IPS observations to set values of Vsw at the outer boundary of the Schatten Current Sheet (SCS) model, which is the outer portion of the WSA model. The common theme of these works is an attempt to use more than only the 1 AU Vsw observations to trace heliospheric magnetic field lines. The use of this additional observational information could serve as the next advance over the simple PS approximation to determine the solar sources of $1 \mathrm{AU}$ magnetic fields.

\section{Conclusion}

Because the solar source regions of SEP events are becoming better defined, it is important to understand and improve on the PS approximation to determine more accurately the coronal or photospheric magnetic footpoints of the SEP observations at L1. We have run the WSA model on 21 selected CRs to compare the WSA CLs with those deduced from the PS approximation using the model Vsw values. The goal is to use results of these model comparisons as a guide for the applicability of the PS approximation with spacecraft data. The comparisons here have shown that the PS approximation agrees well, to within several degrees at the 5 Rs source, with the WSA model for the great majority of the CRs. The PS approximation fails in the SW interaction regions characterized by minimum Vsw values in slow SW regions and subsequent rapid $\mathrm{V}_{\mathrm{sw}}$ increases, the HSE events, marking the onsets of high speed streams. The WSA model results for the interaction regions show that the WSA CLs lie east of the inferred PS CLs by as much as tens of degrees and often in regions of opposite magnetic polarity. We hope that other SW models can confirm or improve on the WSA model results presented here

Acknowledgements. SWK was funded by AFOSR Task 2301RDZ4. DAS was an AFRL Summer Space Scholar.

\section{REFERENCES}

Agueda, N., Klein, K.-L., Vilmer, N., RodríguezGasén, R., Malandraki, O. E., Papaioannou, A., Subirà, M., Sanahuja, B., et al.: 2014, Release timescales of solar energetic particles in the low corona, A\&A, 570:A5, doi: 10.1051/0004-6361/201423549.

Arge, C.N., Luhmann, J.G., Odstrcil, D., Schrijver, C.J., Li, Y.: 2004, Stream structure and coronal sources of the solar wind during the May 12th, 1997 CME, J. Atmosph. Solar-Terres. Phys., 66, 1295, doi:10.1016/j.jastp.2004.03.018.

Arge, C.N., Henney, C.J., Hernandez, I.G., Toussaint, W.A., Koller, J., Godinez, H.C.: 2013, Modeling the corona and solar wind using ADAPT maps that include far-side observations, in SOLAR WIND 13: Proceedings of the Thirteenth International Solar Wind Conference. AIP Conf. Proc. 1539, 11. doi: $10.1063 / 1.4810977$.

Bučík, R., Innes, D. E., Mall, U., Korth, A., Mason, G. M., Gómez-Herrero, R.: 2014 , Multi-spacecraft observations of recurrent $3 \mathrm{He}-$ rich solar energetic particles, ApJ, 786:71, doi: 10.1088/0004-637X/786/1/71.

Chen, N., Bučík, R., Innes, D.E., Mason, G.M.: 2015 , Case studies of multi-day ${ }^{3} \mathrm{He}$-rich solar energetic particle periods, A\&A, 580, A16, doi: http:/10.1051/0004-6361/201525618.

Cohen, C.M.S., Mason, G.M., Mewaldt, R.A., Wiedenbeck, M.E.: 2014, The longitudinal dependence of heavy-ion composition in the 2013 April 11 solar energetic particle event, ApJ, 793:35, doi: 10.1088/0004-637X/793/1/35.

Dresing, N., Gómez-Herrero, R., Klassen, A., Heber, B., Kartavykh, Y., Dröge, W.: 2012, The large longitudinal spread of solar energetic particles during the 17 January 2010 solar event, Sol. Phys., 281, 281-300, doi: 10.1007/s11207-012-0049-y.

Dresing, N., Gómez-Herrero, R., Heber, B., Klassen, A., Malandraki, O., Dröge, W., Kartavykh, Y.: 2014, Statistical survey of widely spread out solar electron events 
observed with STEREO and ACE with special attention to anisotropies, A\&A, 567:A27, doi: http://dx.doi.org/10.1051/0004$6361 / 201423789$.

Gómez-Herrero, R., Dresing, N., Klassen, A., Heber, B., Lario, D., Agueda, N., Malandraki, O.E., Blanco, J.J., Rodrguez-Pacheco, J., Banjac, S.: 2015, Circumsolar energetic particle distribution on 2011 November 3, ApJ, 799:55, doi: 10.1088/0004-637X/799/1/55.

Hickman, K.S., Godinez, H.C., Henney, C.J., Arge, C.N.: 2015, Data assimilation in the ADAPT photospheric flux transport model, Solar Phys., 290, 1105, doi: 10.1007/s112070150666-3.

Jackson, B.V., Hick, P.P., Buffington, A., Yu, H.-S., Bisi, M.M., Tokumaru, M. Zhao, X.: 2015, A Determination of the North-South Heliospheric Magnetic Field Component from Inner Corona Closed-loop Propagation, ApJ, 803, L1, doi: 10.1088/2041-8205/803/1/L1.

Kahler, S.W., Arge, C.N., Akiyama, S., Gopalswamy, N.: 2014, Do solar coronal holes affect the properties of solar energetic particle events?, Sol. Phys., 289, 657, doi:http://dx.doi.org/10.1007/s11207-0130427-0.

Klassen, A., Dresing, N., Gómez-Herrero, Heber, B.: 2015, First simultaneous observations of a near-relativistic electron spike event by both STEREO spacecraft, A\&A, 580, A115, doi: 10.1051/0004-6361/201525700.

Klein, K.-L., Krucker, S., Lointier, G., Kerdraon, A.: 2008, Open magnetic flux tubes in the corona and the transport of solar energetic particles, A\&A, 486, 589, doi: 10.1051/00046361:20079228.

Ko, Y.-K., Tylka, A.J., Ng, C.K., Wang, Y.-M., Dietrich, W.F.: 2013, Source regions of the interplanetary magnetic field and variability in heavy-ion elemental composition in gradual solar energetic particle events, ApJ, 776:92, doi: 10.1088/0004-637X/776/2/92.

Laitinen, T., Dalla, S., Marsh, M.S.: 2013, Energetic particle cross-field propagation early in a solar event, ApJ, 773:L29. doi: 10.1088/20418205/773/2/L29.

Lario, D., Kallenrode, M.-B., Decker, R B., Roelof, E.C., Krimigis, S.M., Aran, A., Sanahuja, B.: 2006, Radial and Longitudinal Dependence of Solar 4-13 MeV and 27-37 MeV Proton Peak Intensities and Fluences: Helios and IMP 8 Observations, ApJ, 653, 1531-1544, doi: $10.1086 / 508982$.

Lario, D., Aran, A., Gómez-Herrero, R., Dresing, N., Heber, B., Ho, G.C., Decker, R.B., Roelof, E.C.: 2013, Longitudinal and Radial Dependence of Solar Energetic Particle Peak Intensities: STEREO, ACE, SOHO, GOES, and MESSENGER Observations, ApJ, 767:41, doi: 10.1088/0004-637X/767/1/41.

Lario, D., Raouafi, N.E., Kwon, R.-Y., Zhang, J., Gómez-Herrero, R., Dresing, N., Riley, P.: 2014, The Solar Energetic Particle Event on 2013 April 11: An Investigation of its Solar Origin and Longitudinal Spread, ApJ, 797:8, doi: 10.1088/0004-637X/797/1/8.

Lee, C.O., Arge, C.N., Odstrčil, D., Millward, G., Pizzo, V., Quinn, J.M., Henney, C.J.: 2013, Ensemble modeling of CME propagation, Sol. Phys., 285, 349368. doi: 10.1007/s11207-012-9980-1.

Lemen, J.R., Title, A.M., Akin, D.J., Boerner, P.F., Chou, C., et al.: 2012, The Atmospheric Imaging Assembly (AIA) on the Solar Dynamics Observatory (SDO), Sol. Phys.275, 17, doi: 10.1007/s11207-011-9776-8.

Li, B., Cairns, I.H., Gosling, J.T., et al.: 2016, Mapping magnetic field lines between the Sun and Earth, J. Geophys. Res., in press.

Linker, J.A.; Miki, Z., Riley, P., Downs, C., Lionello, R., Henney, C., Arge, C.N.: 2013, Coronal and heliospheric modeling using fluxevolved maps, SOLAR WIND 13: Proceedings of the Thirteenth International Solar Wind Conference. AIP Conference Proceedings, 1539, 26-29, doi: 10.1063/1.4810981.

Luhmann, J.G., Li, Y., Riley, P., Arge, C.N., Liu, Y., DeToma, G.: 2008, Challenges created by active regions in global models for space 
weather uses, in ASP Conf. Proc. 383, Subsurface and Atmospheric Influences on Solar Activity ASP Conference Series, Vol. 383, eds R. Howe, R. W. Komm, K. S. Balasubramaniam and G. J. D. Petrie. San Francisco: Astronomical Society of the Pacific, 133.

Luhmann, J.G., Li, Y., Bercik, D.J., Wang, L.,Odstrcil, D.: 2012, Issues in heliospheric field mapping to flare SEP sources, Space Weather: The space radiation environment, AIP Conf. Proc. 1500, 3-13, doi: 10.1063/1/4768737.

MacNeice, P.: 2009, Validation of community models: 2. Development of a baseline using the Wang-Sheeley-Arge model, Space Weather, 7, S12002, doi:10.1029/2009SW000489.

MacNeice, P., Elliott, B., Acebal, A.: 2011, Validation of community models: 3. Tracing field lines in heliospheric models, Space Weather, 9, S10003, doi:10.1029/2011SW000665.

Manoharan, P., Kim, T., Pogorelov, N.V., Arge, C.N., Manoharan, P.K.: 2015, Modeling solar wind with boundary conditions from interplanetary scintillations, Journal of Physics: Conf. Ser., 642, 012016, doi:10.1088/17426596/642/1/012016.

Miteva, R., Klein, K.-L., Malandraki, O., Dorrian, G.: 2013, Solar energetic particle events in the 23rd solar cycle: Interplanetary magnetic field configuration and statistical relationship with flares and CMEs, Sol. Phys., 282, 579-613, doi: 10.1007/s11207-012-0195-2.

Miteva, R., Klein, K.-L., Kienreich, I., Temmer, M., Veronig, A., Malandraki, O.E.: 2014, Solar energetic particles and associated EIT disturbances in solar cycle 23, Sol. Phys., 289, 26012631, doi: 10.1007/s11207-014-0499-5.

Nitta, N.V.: 2012, Magnetic field connection and large scale coronal disturbances in the context of gradual SEP events, PHYSICS OF THE HELIOSPHERE: A 10 YEAR RETROSPECTIVE: Proceedings of the 10th Annual International Astrophysics Conference. AIP Conference Proceedings, 1436, 259-264 doi: $10.1063 / 1.4723617$.

Nitta, N.V., Mason, G.M., Wang, L., Cohen, C.M.S., Wiedenbeck, M.E.: 2015, Solar sources of 3He-rich solar energetic particle events in solar cycle 24, ApJ, 806:235, doi: 10.1088/0004$637 \mathrm{X} / 806 / 2 / 235$

Nitta, N.V., De Rosa, M.L.: 2008, A comparison of solar open field regions found by type III radio bursts and the potential field source surface model, Astrophys. J. Let., 673, L207, doi: $10.1086 / 527548$.

Nolte, J.T., Roelof, E.C.: 1973, Large-scale structure of the interplanetary medium, I: High coronal source longitude of the quiettime solar wind Sol. Phys., 33, 241-257, doi: 10.1007/BF00152395.

Nolte, J.T., Krieger, A.S., Roelof, E.C., Gold, R.E.: 1977, High coronal structure of high velocity solar wind stream sources, Sol. Phys., 51, 459-471, doi: 10.1007/BF00216379.

Norquist, D.C.: 2013, Forecast performance assessment of a kinematic and a magnetohydrodynamic solar wind model, Space Weather, 11, 17, doi: 10.1029/2012SW000853.

Norquist, D.C., Meeks, W.C.: 2010, A comparative verification of forecasts from two operational solar wind models, Space Weather, 8, S12005, doi: 10.1029/2010SW000598.

Odstrcil, D., Pizzo, V.J.: 2009, Numerical heliospheric simulations as assisting tool for interpretation of observations by STEREO Heliospheric Imagers, Sol. Phys., 259, 297-309, doi: 10.1007/s11207-009-9449-z.

Owens, M.J., Spence, H.E., McGregor, S., Hughes, W.J., Quinn, J M., Arge, C.N., Riley, P., Linker, J., Odstrcil, D.: 2008, Metrics for solar wind prediction models: Comparison of empirical, hybrid, and physics-based schemes with 8 years of L1 observations, Space Weather, 6, S08001, doi: 10.1029/2007SW000380

Owens, M.J., Forsyth, R.J.: 2013, The Heliospheric Magnetic Field, Living Rev. Solar Phys., 10:5, doi: 10.12942/lrsp-2013-5.

Park, J., Innes, D.E., Bucik, R., Moon, Y.J.: 2013, The Source Regions of Solar Energetic Particles Detected by Widely Separated 
Spacecraft, ApJ, 779:184, doi: 10.1088/0004$637 \mathrm{X} / 779 / 2 / 184$.

Park, J., Innes, D.E., Bucik, R., Moon, Y.J., Kahler, S.W.: 2015, Study of solar energetic particle associations with coronal extreme-ultraviolet waves, ApJ, 808:3, doi: $10.1088 / 0004-637 \mathrm{X} / 808 / 1 / 3$.

Prise, A.J., Harra, L.K., Matthews, S.A., Long, D.M., Aylward, A D.: 2014, An investigation of the CME of 3 November 2011 and its associated widespread solar energetic particle event, Sol. Phys., 289, 1731-1744, doi: 10.1007/s11207-013-0435-0.

Ragot, B.R.: 2011, Nonlinear multiscale simulation of turbulent flux tubes, ApJ, 740:119, doi: 10.1088/0004-637X/740/2/119.

Ragot, B.R.: 2012, Nonresonant interaction of charged energetic particles with low-frequency noncompressive turbulence: numerical simulation, ApJ, 758:89, doi: 10.1088/0004$637 \mathrm{X} / 758 / 2 / 89$.

Reames, D.V.: 2013, The two sources of solar energetic particles, Space Sci. Rev., 175, 53, doi: 10.1007/s11214-013-9958-9.

Richardson, I.G.: 2004, Energetic particles and corotating interaction regions in the solar wind, Space Science Rev., 111, 267, doi: 10.1023/B:SPAC.0000032689.52830.3e.

Richardson, I.G., and Cane, H.V.: 2012, Near-earth solar wind flows and related geomagnetic activity during more than four solar cycles (1963-2011), J. Space Weather Space Clim., 2, A02, doi: http://dx.doi.org/10.1051/swsc/2012003.

Riley, P., Lionello, R.: 2011, Mapping solar wind streams from the Sun to 1 AU: A comparison of techniques, Sol. Phys., 270, 575, doi: 10.1007/s11207-011-9766-x.

Richardson, I.G., von Rosenvinge, T.T., Cane, H.V., Christian, E.R., Cohen, C.M.S., Labrador, A.W., Leske, R.A., Mewaldt, R.A., Wiedenbeck, M.E., Stone, E.C.: 2014, > 25 $\mathrm{MeV}$ proton events observed by the high energy telescopes on the STEREO A and B spacecraft and/or at Earth during the first $\sim$ seven years of the STEREO Mission, Solar Phys., 289, 3059, doi: 10.1007/s11207-014-0524-8.

Rouillard, A.P., Odstřcil, D., Sheeley, N.R., Tylka, A., Vourlidas, A., Mason, G., Wu, C.-C., Savani, N.P., Wood, B.E., Ng, C.K., Stenborg, G., Szabo, A., St. Cyr, O.C.: 2011, Interpreting the properties of solar energetic particle events by using combined imaging and modeling of interplanetary shocks, ApJ, 735:7, doi: 10.1088/0004-637X/735/1/7.

Rouillard, A.P., Sheeley, N.R., Tylka, A., Vourlidas, A., Ng, C.K., Rakowski, C., Cohen, C M. S., Mewaldt, R.A., Mason, G.M., Reames, D., et al.: 2012, The longitudinal properties of a solar energetic particle event investigated using modern solar imaging, ApJ, 752:44, doi: 10.1088/0004-637X/752/1/44.

Schatten, K.H.: 1972, Current sheet magnetic model for the solar corona, Solar Wind, eds Sonnet, C.P., Coleman, P.J., and Wilcox, J.M., NASA, 44-54.

Schulte in den Bäumen, H., Cairns, I.H., Robinson, P.A.: 2012, Nonzero azimuthal magnetic fields at the solar source surface: Extraction, model, and implications, J. Geophys. Res., 117, A10104, doi:10.1029/2012JA017705.

Shen, C., Wang, Y., Ye, P., Wang, S.: 2006, Is there any evident effect of coronal holes on gradual solar energetic particle events? ApJ, 639, 510-515. doi: 10.1086/499199.

Shen, C.-L., Yao, J., Wang, Y.-M., Ye, P.-Z., Zhao, X.-P., Wang, S.: 2010, Influence of coronal holes on CMEs in causing SEP events, Res. Astron. Astrophys., 10, 1049-1060. doi: 10.1088/1674-4527/10/10/008.

Wiedenbeck, M.E., Mason, G.M., Cohen, C.M.S., Nitta, N.V., Gmez-Herrero, R., Haggerty, D.K.: 2013, Observations of solar energetic particles from $3 \mathrm{He}$-rich events over a wide range of heliographic longitude, ApJ, 762:54, doi: 10.1088/0004-637X/762/1/54.

Wiedenbeck, M.E., Cohen, C.M.S., Klassen, A., Leske, R.A., Liewer, P.A., Mason, G.M., Nitta, 
N.: 2015, Constraints on mechanisms for longitudinal spreading of impulsive SEPs from multispacecraft observations of scatter-free events, Proc. 34th ICRC, PoS 106.

This 2-column preprint was prepared with the AAS LATEX macros v5.2. 
Table 1: CRs and High Speed Events with $-($ WSA-PS $) \geq \Delta 15^{\circ}$.

\begin{tabular}{|c|c|c|c|c|c|}
\hline CR \# & Start Date & SEP/ICME ${ }^{\mathrm{a}}$ & $\Delta \mathrm{Vsw}^{\mathrm{b}}$ & $\Delta \mathrm{L}^{\mathrm{c}}$ & Polarity \\
\hline \multirow[t]{3}{*}{2094} & 27 Feb 2010 & $0 / 0$ & 162 & $30^{\circ}$ & $\mathrm{P}$ \\
\hline & & & 140 & $17^{\circ}$ & $\mathrm{P}$ \\
\hline & & & 200 & $25^{\circ}$ & $\mathrm{P}$ \\
\hline \multirow[t]{3}{*}{2096} & 22 Apr 2010 & $0 / 0$ & 043 & $15^{\circ}$ & $\mathrm{N}$ \\
\hline & & & 212 & $20^{\circ}$ & $\mathrm{P}$ \\
\hline & & & 237 & $25^{\circ}$ & $\mathrm{P}$ \\
\hline \multirow[t]{3}{*}{2098} & 16 Jun 2010 & $0 / 0$ & 115 & $20^{\circ}$ & $\mathrm{N}$ \\
\hline & & & 288 & $30^{\circ}$ & $\mathrm{N}$ \\
\hline & & & 118 & $21^{\circ}$ & $\mathrm{N}$ \\
\hline \multirow[t]{3}{*}{2100} & 9 Aug 2010 & $4 / 0$ & 121 & $25^{\circ}$ & $\mathrm{N}$ \\
\hline & & & 188 & $33^{\circ}$ & $\mathrm{N} / \mathrm{P}$ \\
\hline & & & 165 & $20^{\circ}$ & $\mathrm{N}$ \\
\hline \multirow{4}{*}{2102} & 3 Oct 2010 & $0 / 0$ & 153 & $35^{\circ}$ & $\mathrm{P}$ \\
\hline & & & 109 & $20^{\circ}$ & $\mathrm{P}$ \\
\hline & & & 164 & $25^{\circ}$ & $\mathrm{P}$ \\
\hline & & & 257 & $25^{\circ}$ & $\mathrm{N}$ \\
\hline \multirow[t]{2}{*}{2104} & 26 Nov 2010 & $0 / 0$ & -18 & $22^{\circ}$ & $\mathrm{P}$ \\
\hline & & & 214 & $25^{\circ}$ & $\mathrm{P}$ \\
\hline \multirow[t]{2}{*}{2105} & 24 Dec 2010 & $1 / 0$ & 318 & $37^{\circ}$ & $\mathrm{P}$ \\
\hline & & & 294 & $32^{\circ}$ & $\mathrm{P}$ \\
\hline \multirow[t]{3}{*}{2106} & 20 Jan 2011 & $6 / 0$ & 194 & $37^{\circ}$ & $\mathrm{P}$ \\
\hline & & & 185 & $20^{\circ}$ & $\mathrm{N}$ \\
\hline & & & 269 & $27^{\circ}$ & $\mathrm{N} / \mathrm{P}$ \\
\hline 2115 & 22 Sep 2011 & $2 / 1$ & $\ldots$ & $\ldots$ & $\ldots$ \\
\hline \multirow[t]{4}{*}{2118} & 13 Dec 2011 & $5 / 0$ & 152 & $27^{\circ}$ & $\mathrm{N}$ \\
\hline & & & 112 & $25^{\circ}$ & $\mathrm{P}$ \\
\hline & & & 340 & $27^{\circ}$ & $\mathrm{P}$ \\
\hline & & & 283 & $25^{\circ}$ & $\mathrm{N}$ \\
\hline \multirow[t]{3}{*}{2119} & 9 Jan 2012 & $4 / 2$ & 077 & $25^{\circ}$ & $\mathrm{P}$ \\
\hline & & & 296 & $35^{\circ}$ & $\mathrm{P}$ \\
\hline & & & 247 & $27^{\circ}$ & $\mathrm{P} / \mathrm{N}$ \\
\hline \multirow[t]{3}{*}{2121} & 4 Mar 2012 & $3 / 3$ & 245 & $32^{\circ}$ & $\mathrm{P}$ \\
\hline & & & 084 & $25^{\circ}$ & $\mathrm{P}$ \\
\hline & & & 106 & $22^{\circ}$ & $\mathrm{P}$ \\
\hline \multirow[t]{3}{*}{2123} & 28 Apr 2012 & $3 / 1$ & 105 & $27^{\circ}$ & $\mathrm{P} / \mathrm{N}$ \\
\hline & & & 253 & $30^{\circ}$ & $\mathrm{N}$ \\
\hline & & & 218 & $28^{\circ}$ & $\mathrm{N} / \mathrm{P}$ \\
\hline \multirow[t]{4}{*}{2125} & 21 Jun 2012 & $6 / 3$ & 227 & $18^{\circ}$ & $\mathrm{N}$ \\
\hline & & & 259 & $35^{\circ}$ & $\mathrm{N} / \mathrm{P}$ \\
\hline & & & 031 & $18^{\circ}$ & $\mathrm{P} / \mathrm{N}$ \\
\hline & & & 089 & $16^{\circ}$ & $\mathrm{N}$ \\
\hline \multirow{4}{*}{2126} & $18 \mathrm{Jul} 2012$ & $3 / 1$ & 147 & $15^{\circ}$ & $\mathrm{P}$ \\
\hline & & & 148 & $20^{\circ}$ & $\mathrm{N}$ \\
\hline & & & 127 & $20^{\circ}$ & $\mathrm{N}$ \\
\hline & & & 074 & $16^{\circ}$ & $\mathrm{N}$ \\
\hline \multirow[t]{2}{*}{2135} & 21 Mar 2013 & $1 / 1$ & 260 & $27^{\circ}$ & $\mathrm{N}$ \\
\hline & & & 235 & $27^{\circ}$ & $\mathrm{P}$ \\
\hline
\end{tabular}




\begin{tabular}{cccccc} 
CR \# & Start Date & SEP/ICME & $\Delta$ Vsw $^{\mathrm{b}}$ & $\Delta \mathrm{L}^{\mathrm{c}}$ & Polarity \\
\hline 2141 & 31 Aug 2013 & $1 / 0$ & 089 & $20^{\circ}$ & $\mathrm{P}$ \\
& & & 213 & $20^{\circ}$ & $\mathrm{N}$ \\
2142 & 28 Sep 2013 & $3 / 2$ & 125 & $25^{\circ}$ & $\mathrm{N} / \mathrm{P}$ \\
& & & 165 & $22^{\circ}$ & $\mathrm{P} / \mathrm{N}$ \\
2145 & \multirow{2}{*}{ 19 Dec 2013 } & $3 / 1$ & 190 & $17^{\circ}$ & $\mathrm{P}$ \\
& & & 241 & $22^{\circ}$ & $\mathrm{N}$ \\
2154 & 21 Aug 2014 & $1 / 1$ & 210 & $32^{\circ}$ & $\mathrm{P}$ \\
& & & 109 & $38^{\circ}$ & $\mathrm{N} / \mathrm{P}$ \\
2160 & \multirow{2}{*}{ 1 Feb 2015 } & $1 / 0$ & 232 & $20^{\circ}$ & $\mathrm{P}$ \\
& & & 118 & $19^{\circ}$ & $\mathrm{N} / \mathrm{P}$ \\
\hline \hline
\end{tabular}

${ }^{a}$ Numbers of SEP events and ICMEs during the CR.

${ }^{b}$ WSA forecast increase in Vsw in $\mathrm{km} \mathrm{s}^{-1}$ for each HSE.

$c_{-}$(WSA-PS) in degrees. In all cases the PS longitude lies west of the WSA longitude. 

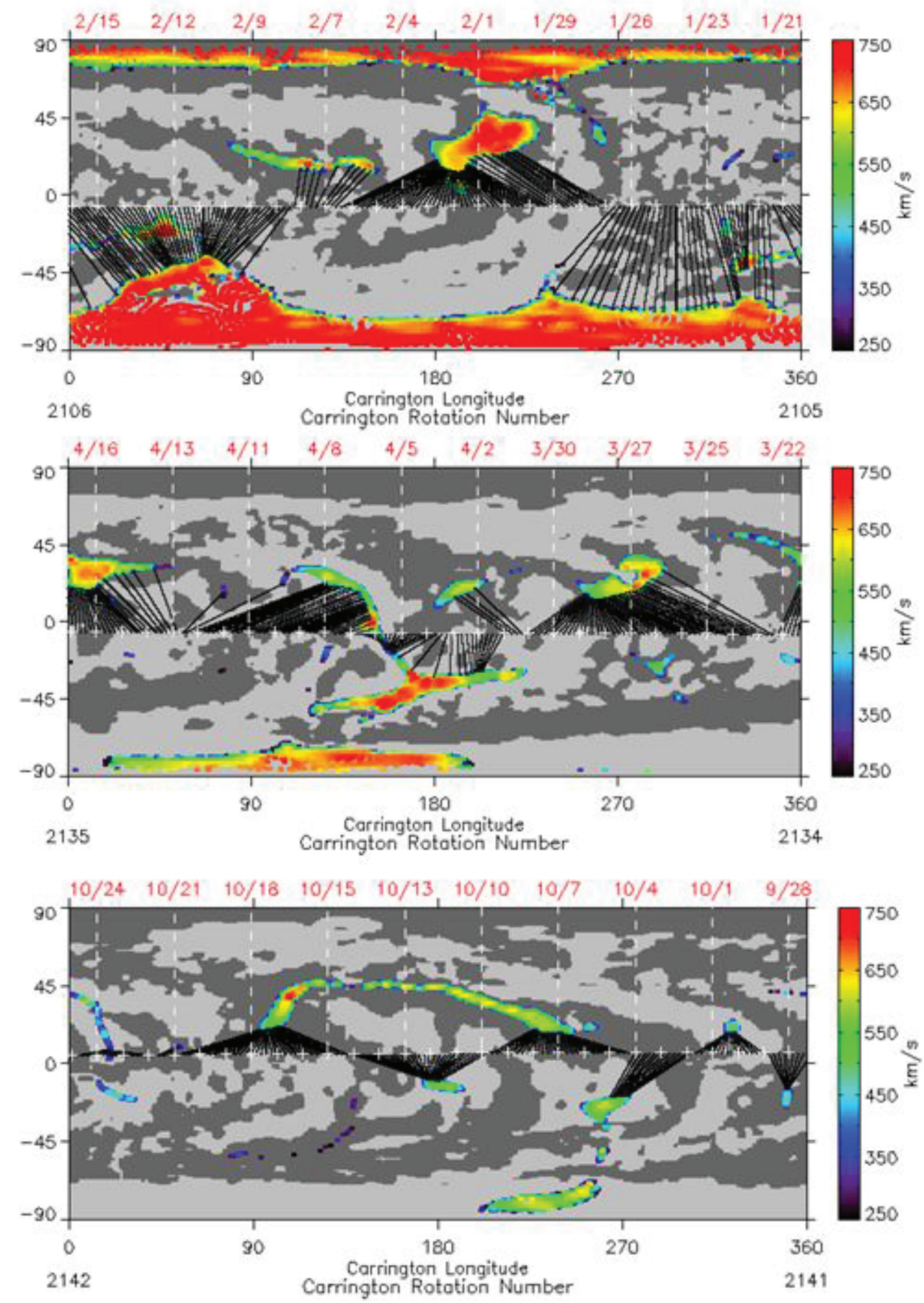

Fig. 1.- NSO GONG synoptic maps of three CRs of Table 1 with derived CHs color-coded for SW speeds. Gray areas are closed fields. Diagonal lines show the magnetic connections from the sub-Earth points on the $5 \mathrm{R}_{\odot}$ outer coronal boundaries to the photospheric CHs at the dates at the top of each map. From top to bottom the maps show predominately high latitude, mid-latitude and low latitude photospheric connections. 


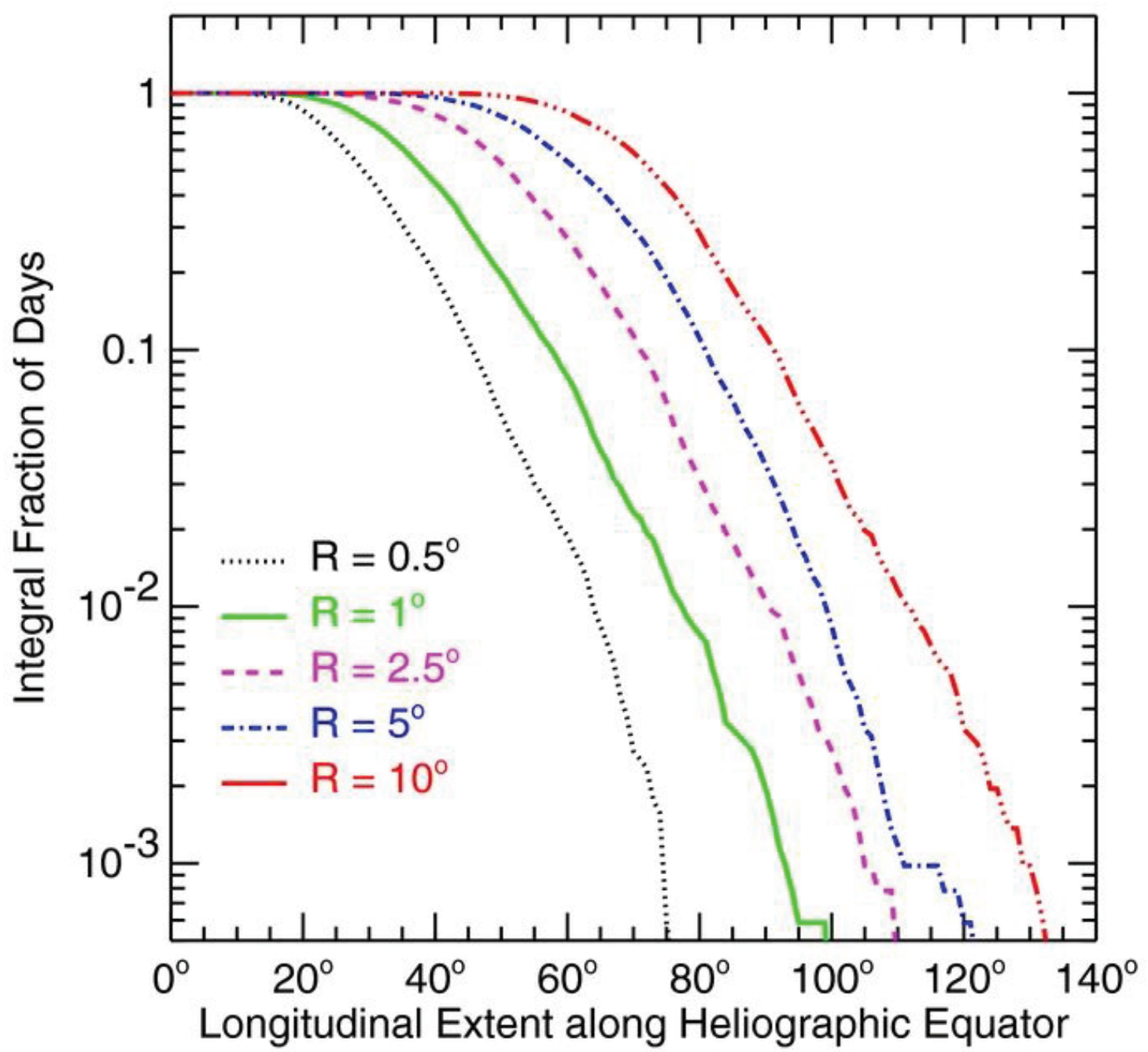

Fig. 2.- Integral distributions of longitudinal size, in number of degrees along the heliographic equator, for the largest cluster of field lines identified in PFSS model maps for each day over a 14 year period extending from 1998 through 2011. Here, a cluster is defined as consisting of those field lines originating within a radius $\mathrm{R}$ at the solar photosphere and mapping to the heliographic equator on the source surface. Curves show the fraction of days when the largest longitudinal spread was greater than the value given by the abscissa. Curves correspond to five different criteria for the cluster radius at the photosphere, as indicated in the legend. From Wiedenbeck et al. (2013). 

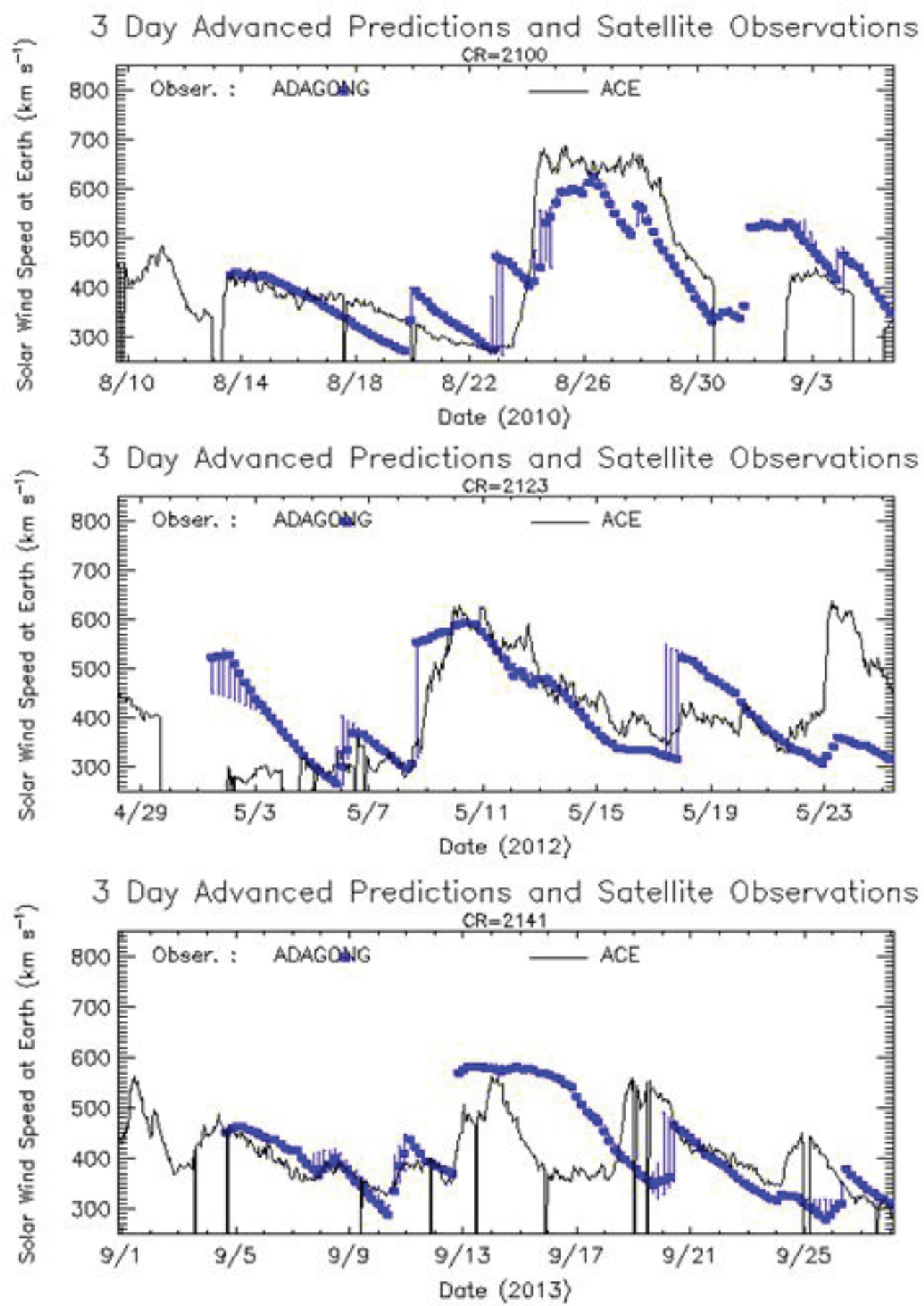

Fig. 3. - Three CRs of Table 1 showing Vsw at L1 from ACE (black profiles) and the WSA forecast values (blue squares) from GONG magnetograms. The times of rapid increases of Vsw are the high-speed events (HSEs) 


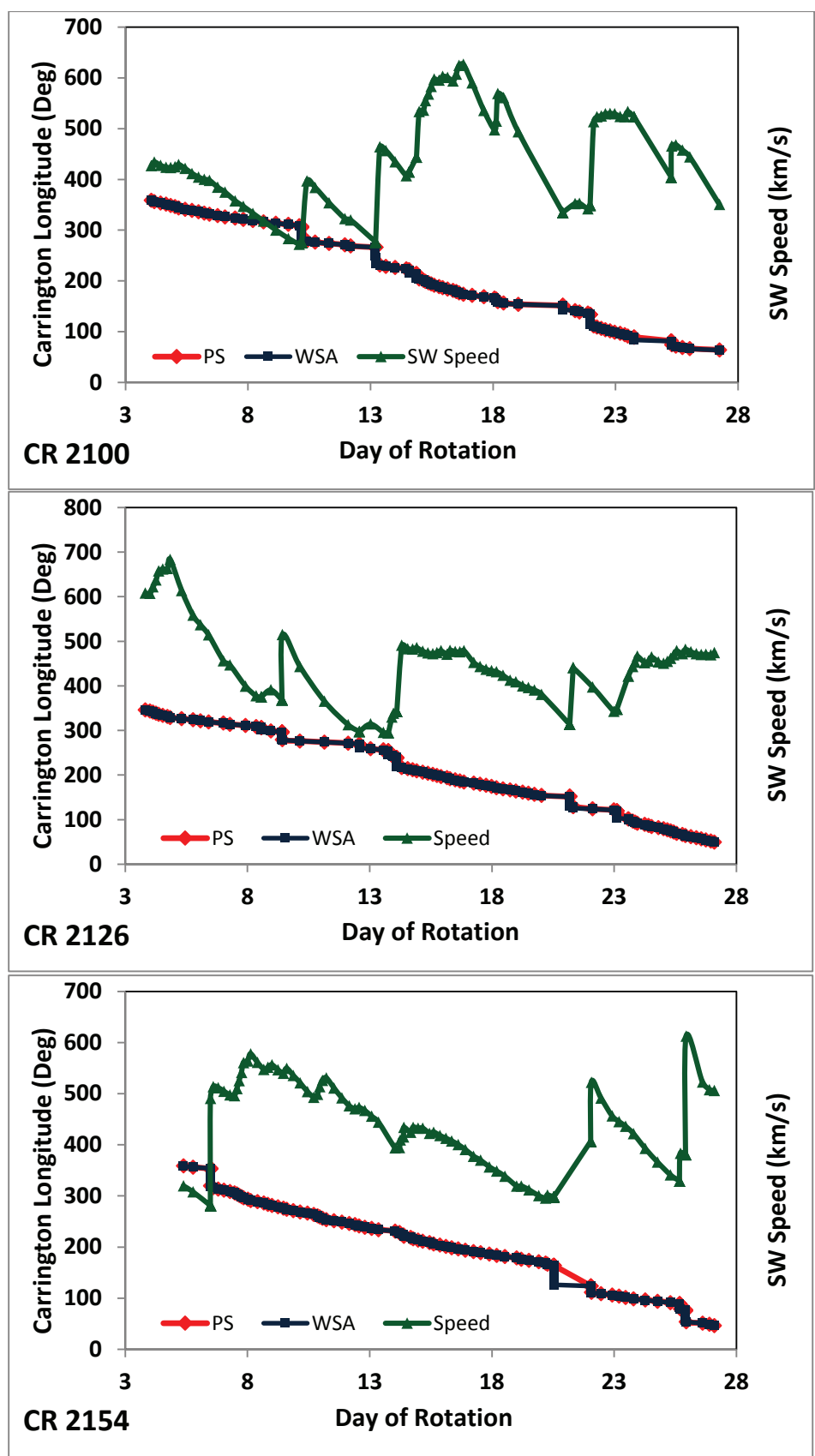

Fig. 4.- Three CRs of Table 1 showing the WSA Vsw at L1 (green profiles), the Carrington longitudes (CLs) of the WSA sources (black profiles), and PS sources based on Vsw (red profiles). 


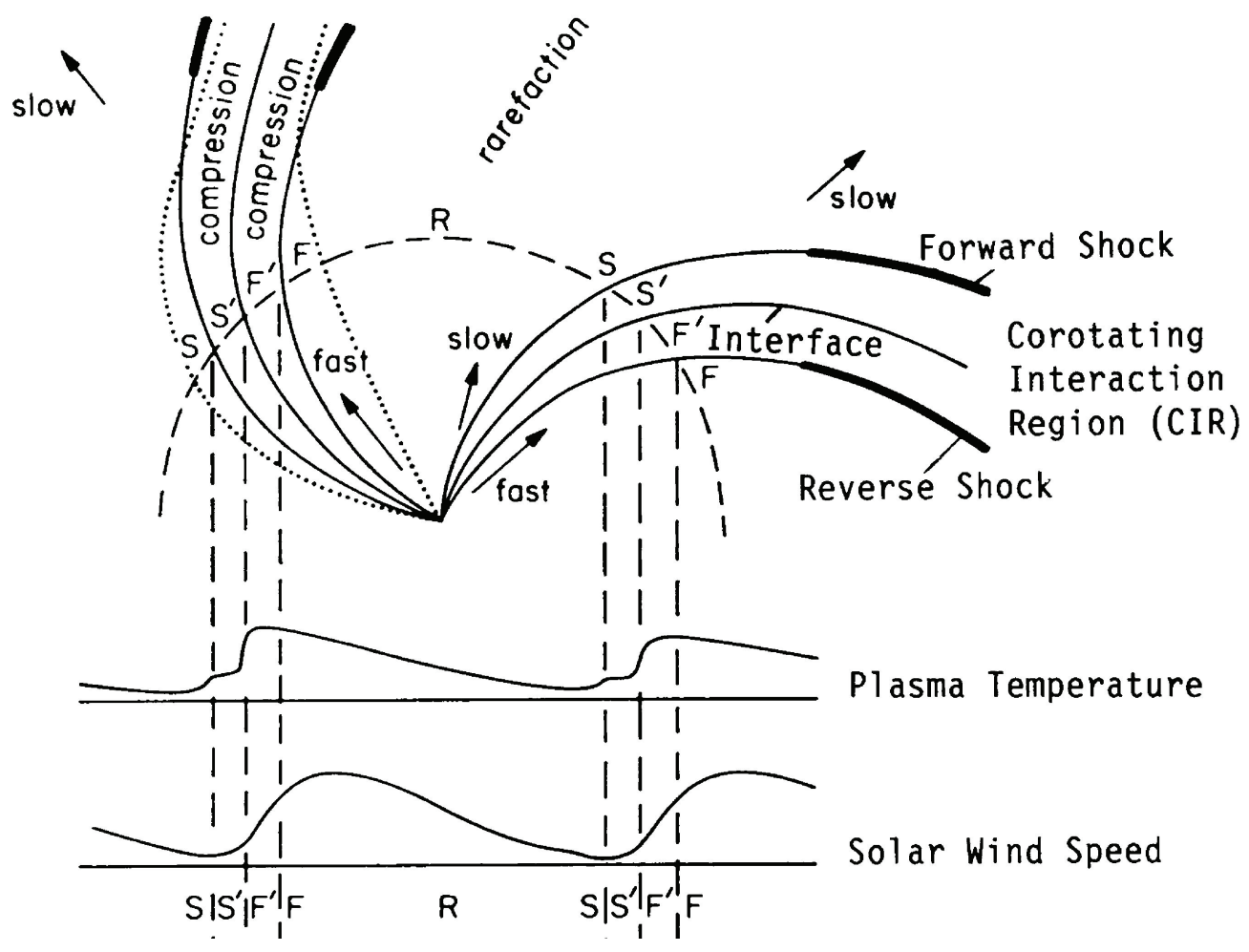

Fig. 5.- Schematic of fast and slow solar wind flows from Richardson (2004). The compression regions at L1 result from the interactions of fast wind flows following slow wind, and the rarefaction regions are due to slow wind flows. 

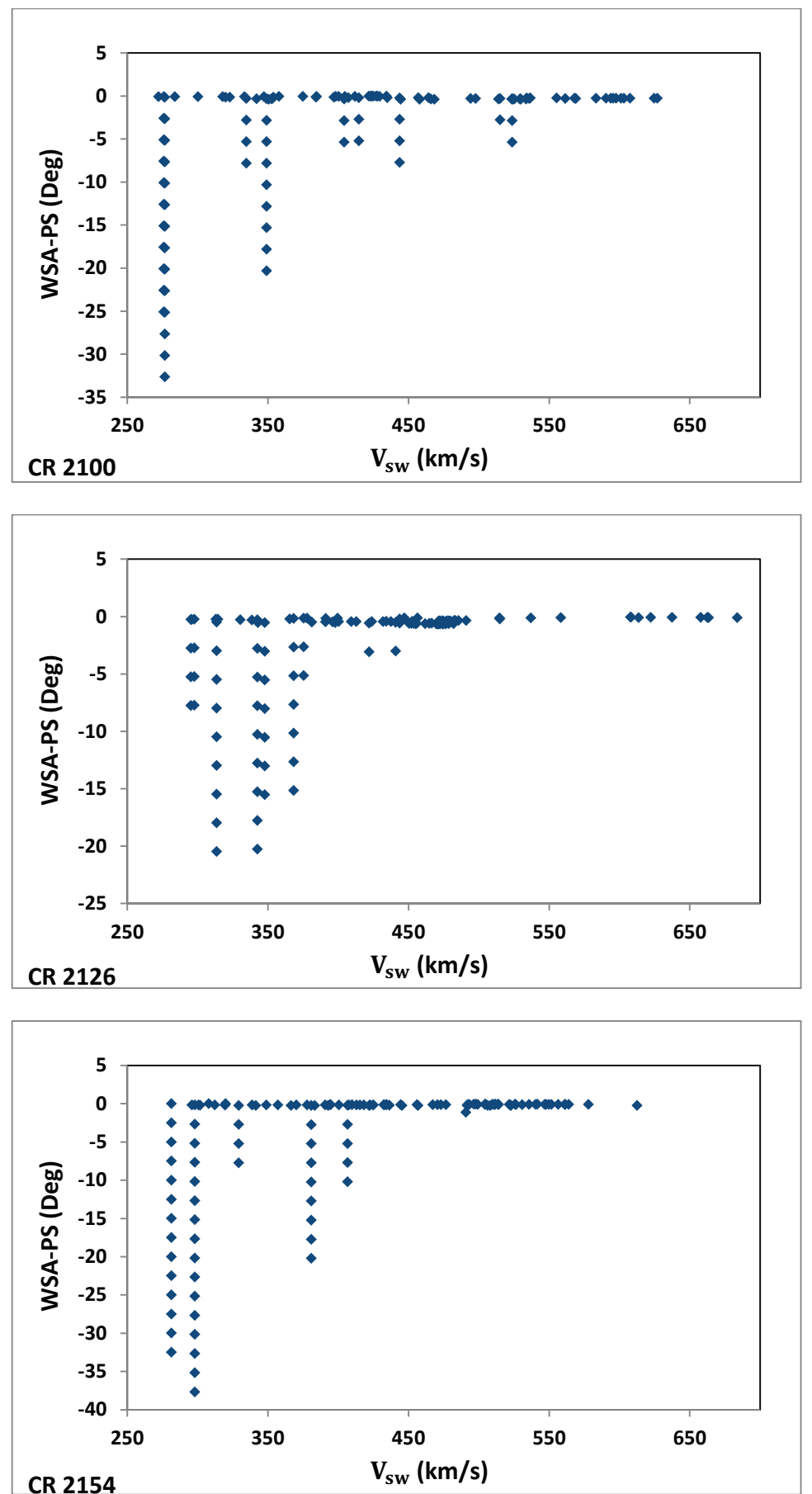

Fig. 6. - Three CR plots of the $\Delta \mathrm{L}=(\mathrm{WSA}-\mathrm{PS})$ versus Vsw from the WSA model at L1. The vertical columns of data points are the bunches with constant Vsw preceding HSEs. The PS CLs remain constant while WSA CLs move eastward, producing increasingly negative values of $\Delta \mathrm{L}$. We select those bunches with $\Delta \mathrm{L}$ reaching $-15^{\circ}$ as our cases of HSEs listed in Table 1 . These are the same CRs as in Figure 4. 

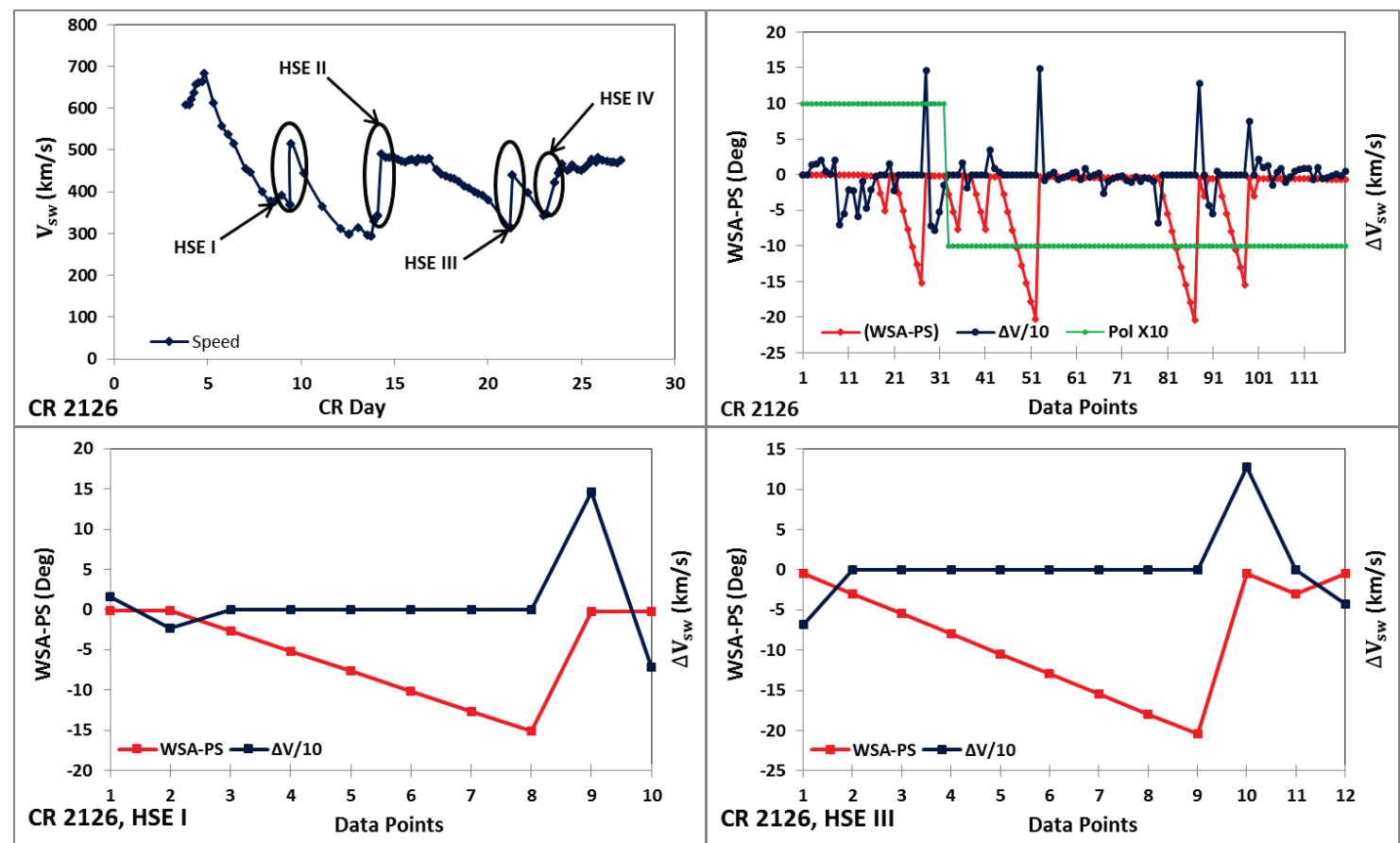

Fig. 7.- Top left panel is the Vsw time profile of CR 2126 (from Figure 4) showing the four HSEs listed in Table 1. Upper right panel shows $\Delta \mathrm{Vsw}$ (black points and lines; $10 \times$ left hand scale) and $\Delta \mathrm{L}$ (red points and lines) for the sequential WSA data points, which form time bunches in the first panel. The WSA CLs move to lower (more eastern) values through the sequences, but the inferred PS CLs remain fixed due to the fixed values of $\mathrm{Vsw}$ at those times. The $\Delta \mathrm{L}$ values reset to $0^{\circ}$ whenever $\Delta \mathrm{Vsw} \neq 0$. We take the maximum deviations as upper limits to the differences between WSA and PS CLs. Bottom panels show HSEs I and III on expanded scales. 

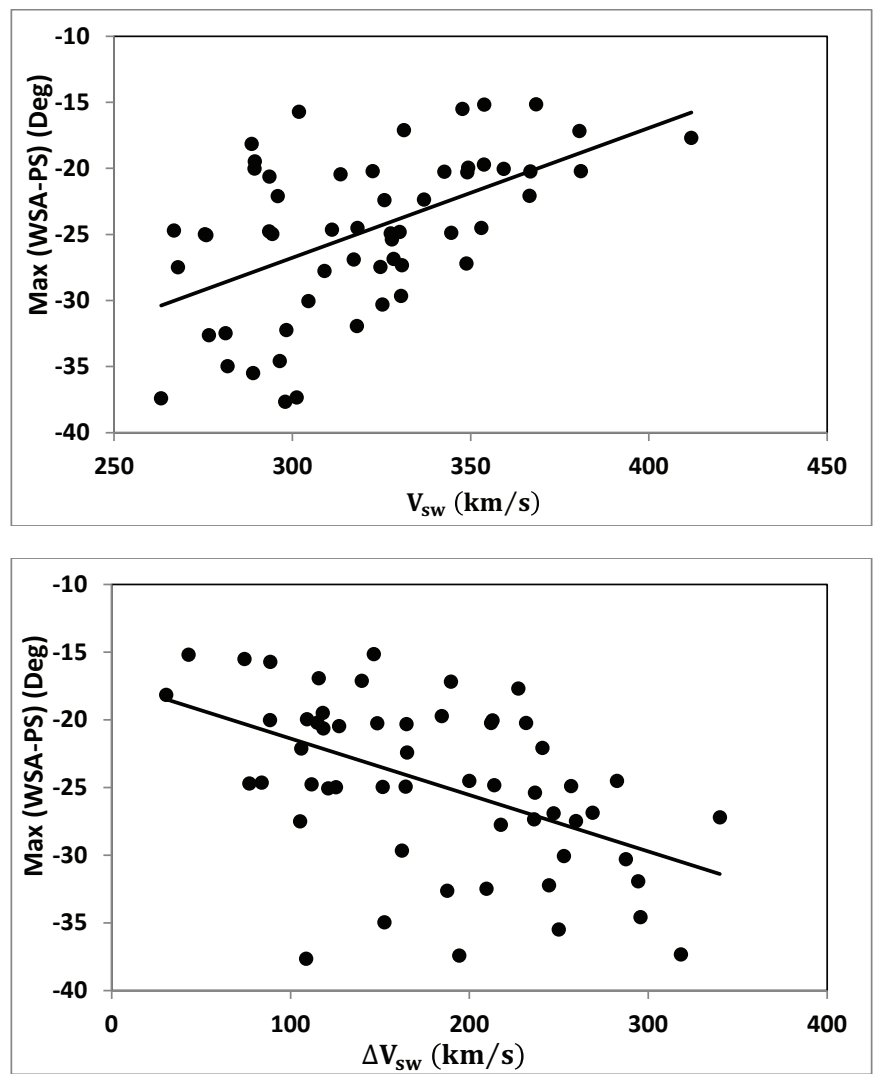

Fig. 8.- Top: Scatter plot of $\Delta \mathrm{L}$ versus Vsw for the constant speeds preceding the HSEs of Table 1. Bottom: plot of the maximum $\Delta \mathrm{L}$ versus associated $\Delta \mathrm{Vsw}$ for 58 HSEs of Table 1 . Strong correlations in both plots are indicated by the least-squares best-fit lines. $\Delta \mathrm{Vsw}>100 \mathrm{~km} \mathrm{~s}^{-1}$ corresponds to the HSE definition of Owens et al. (2008). 

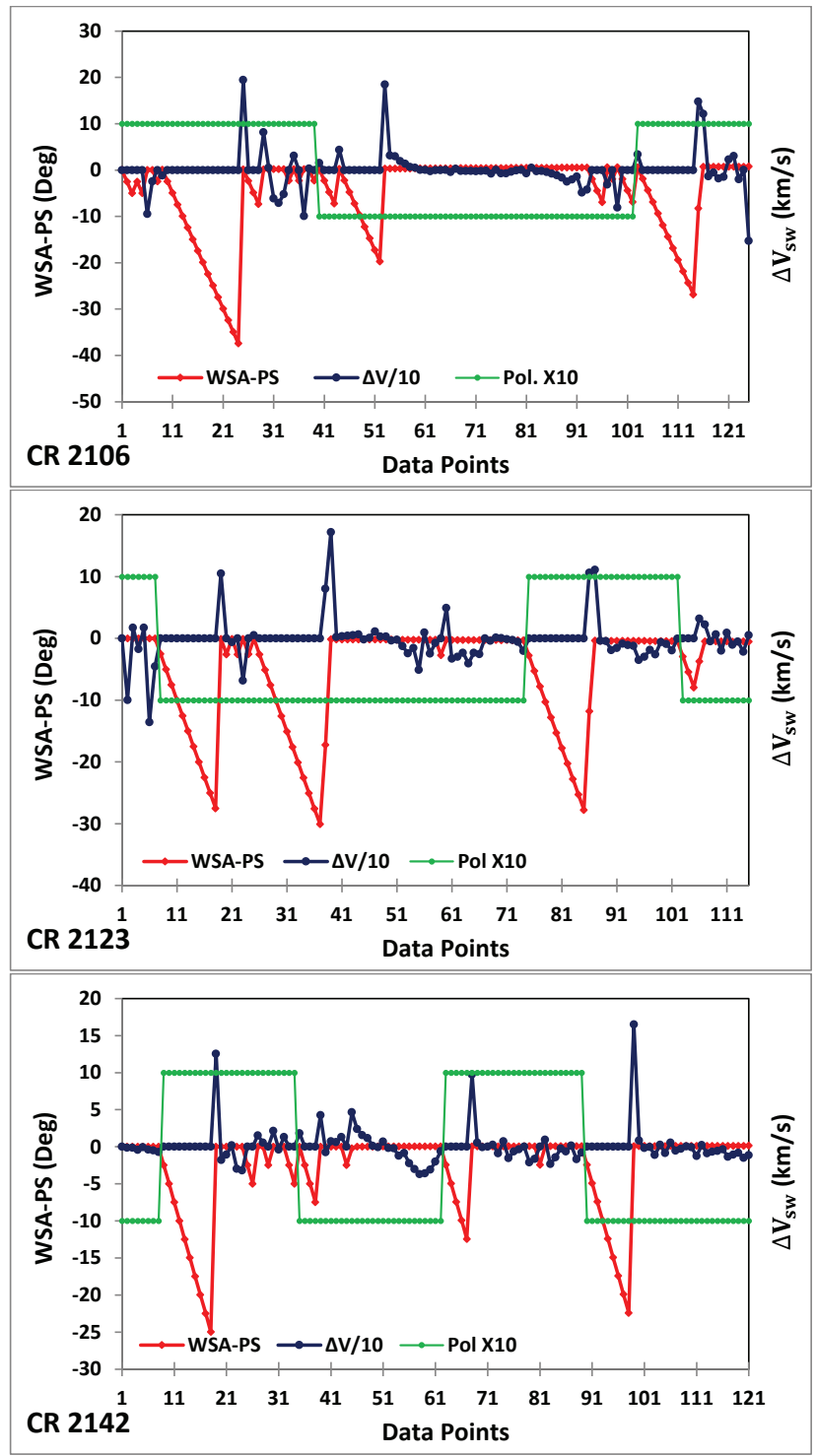

Fig. 9.- WSA data point plots of $\Delta \mathrm{L}=(\mathrm{WSA}-\mathrm{PS})$ (red points) for three CRs showing the magnetic polarities (blue points, indicated as +10 or -10 ) and $\Delta$ Vsw changes (black points). Polarity changes are seen before the HSEs (WSA-PS) $\leq 15^{\circ}$ in each of these rotations, and are also apparent before some smaller $\Delta \mathrm{L}$ excursions (e.g., point 38 in $\mathrm{CR} 2106$ and point 62 in CR 2142). 


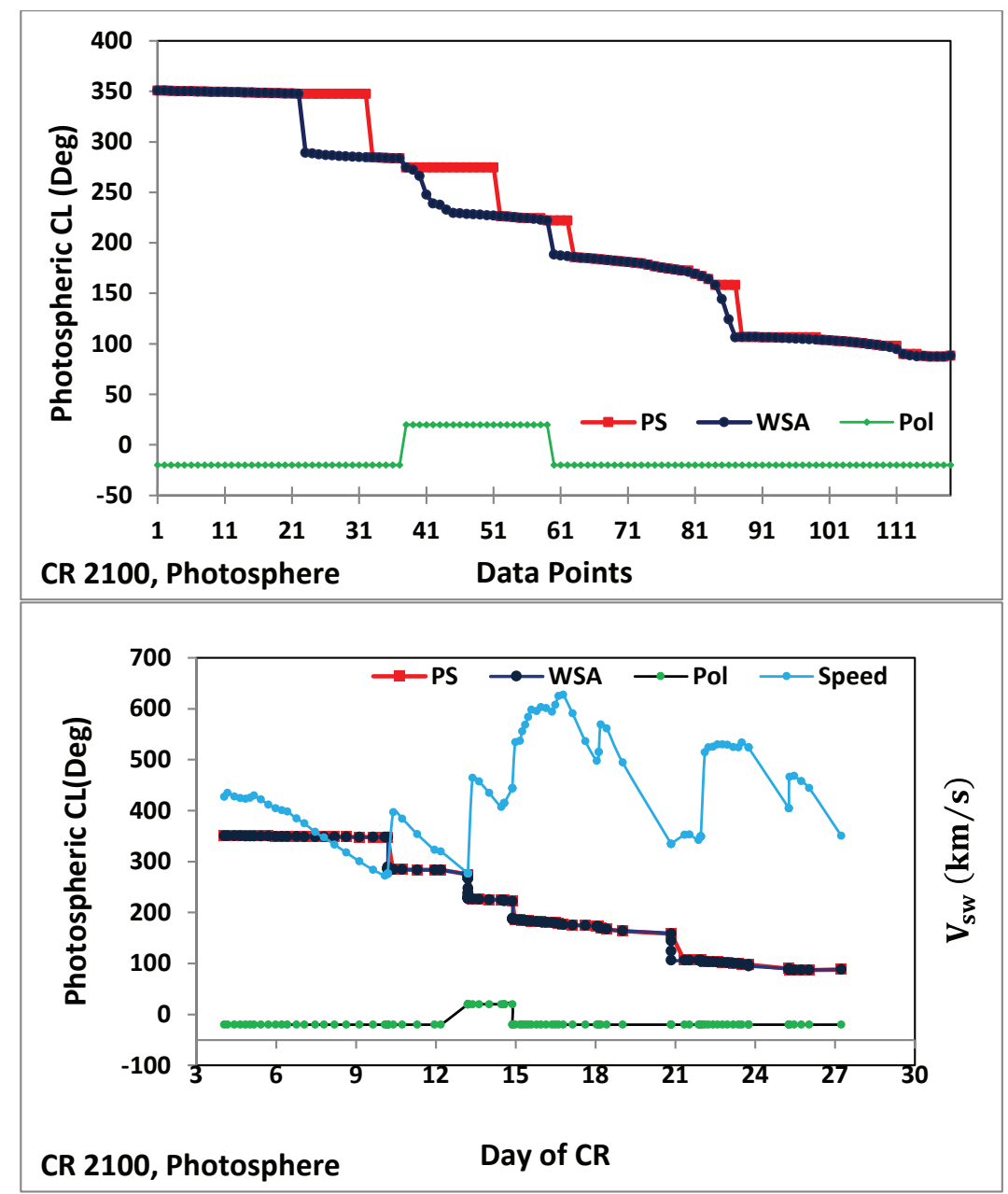

Fig. 10.- Top: Photospheric Carrington longitude (CLp) for WSA (black points) and PS (red points) for CR 2100 as functions of WSA data points. The sharp drops in the WSA CLp occur during the data point bunches at the boundaries of CHs. Magnetic polarities at bottom of the panel are positive or negative (green points). Bottom: The same CR plot as a function of time in the CR and including Vsw. The large values of $\Delta \mathrm{Lp}$ occur just preceding the HSEs. 


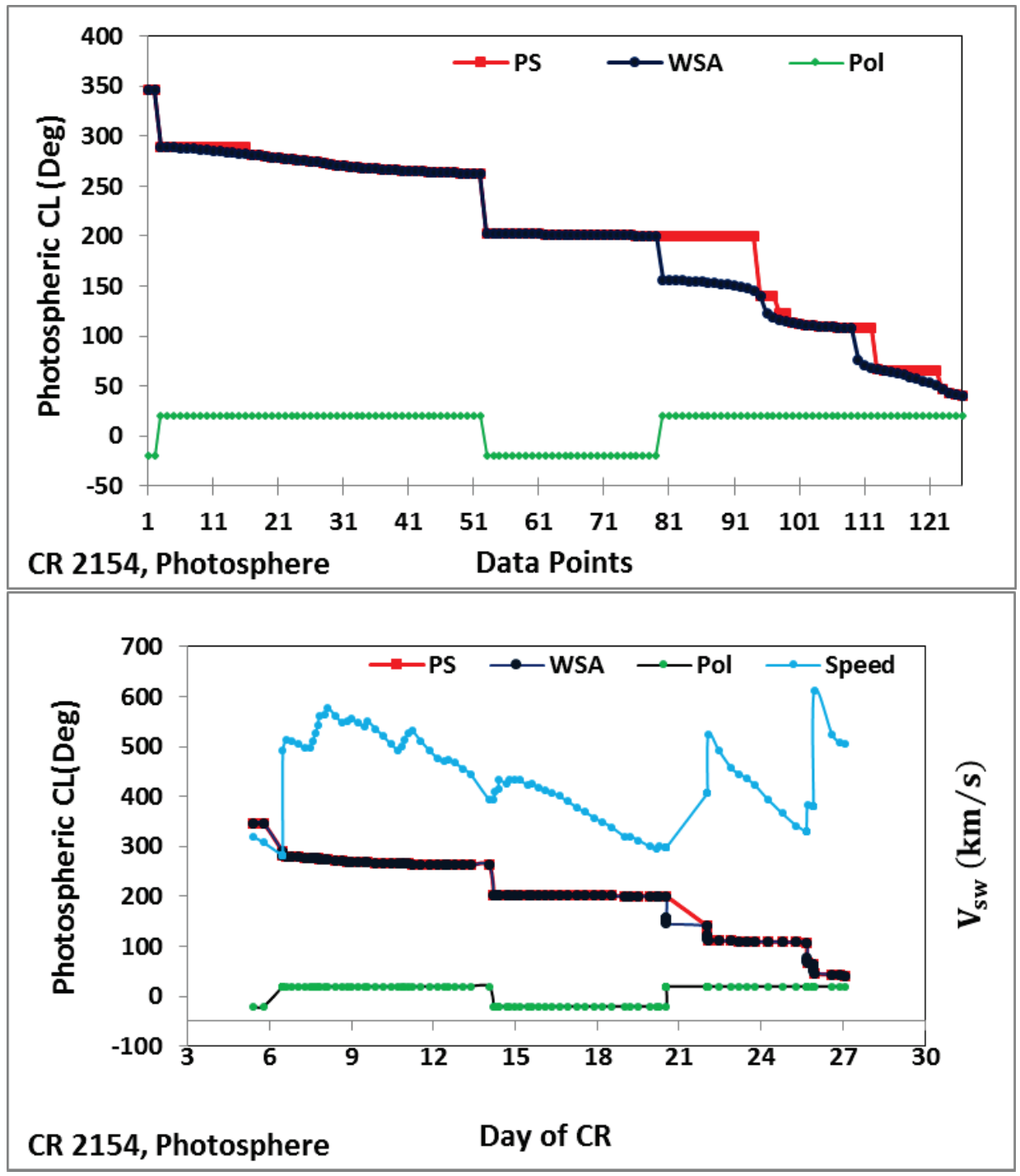

Fig. 11. - The same format as Figure 10, but for CR 2154. 

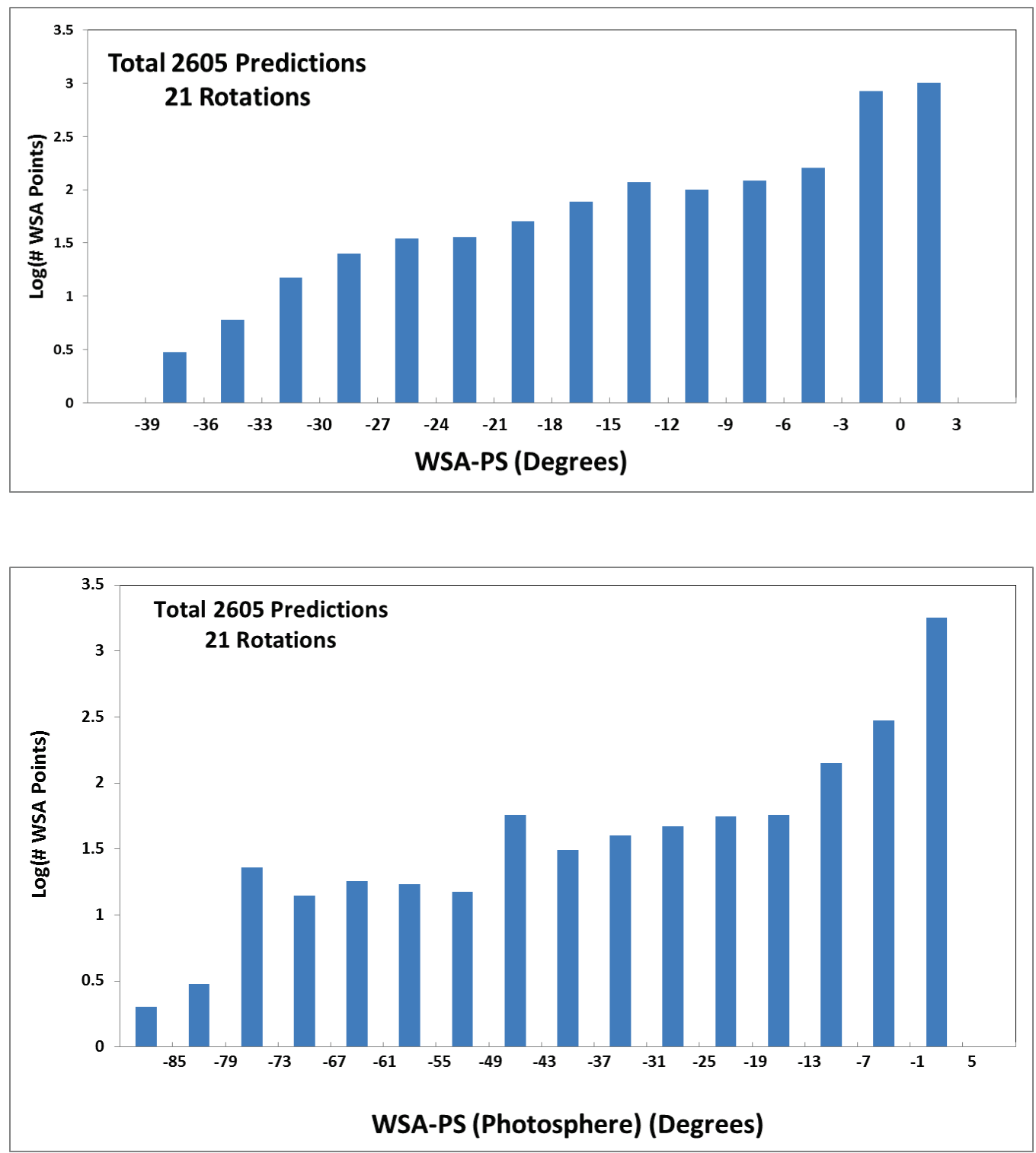

Fig. 12.- Histogram comparison of the $\Delta \mathrm{L}=$ WSA-PS CLs at 5 Rs (top) and at the 1 Rs photosphere $(\Delta \mathrm{Lp})$ (bottom). Each point of the log scale is a single WSA data point from each of the $21 \mathrm{CRs}$ of the study, and the predominately negative values mean that the PS CLs and CLps are west (more positive) of the WSA CLs and CLps in both cases. 